\title{
1 Identification of green lineage osmotic stress pathways
}

2

3 Josep Vilarrasa-Blasi ${ }^{1,2, \bigotimes}$, Tamara Vellosillo, ${ }^{1,2}$, Robert E. Jinkerson ${ }^{2,3}$, Friedrich Fauser ${ }^{2,6}$, 4 Tingting Xiang ${ }^{2,4}$, Benjamin B. Minkoff ${ }^{5}$, Lianyong Wang ${ }^{6}$, Kiril Kniazev ${ }^{1}$, Michael Guzman²,

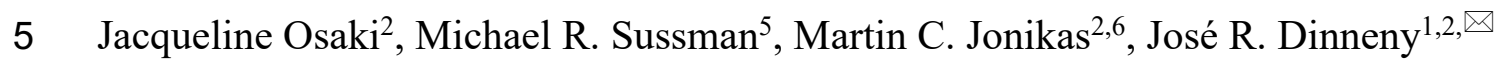

6

7

8 1. Department of Biology, Stanford University, Stanford, CA 94305, USA.

9 2. Department of Plant Biology, Carnegie Institution for Science, CA 94305, USA.

$24 \rrbracket_{\text {e-mail: pep@stanford.edu, dinneny@stanford.edu. }}$ 
Maintenance of water homeostasis is a fundamental cellular process required by all living organisms. Here, we use the green alga Chlamydomonas reinhardtii to establish a foundational understanding of evolutionarily conserved osmotic-stress signaling pathways in the green lineage through transcriptomics, phosphoproteomics, and functional genomics approaches. Five genes acting across diverse cellular pathways were found to be essential for osmotic-stress tolerance in Chlamydomonas including cytoskeletal organization, potassium transport, vesicle trafficking, mitogen-activated protein kinase and chloroplast signaling. We show that homologs of these genes in the multicellular land plant Arabidopsis thaliana have conserved functional roles in stress tolerance and reveal a novel PROFILIN-dependent actin remodeling stage of acclimation that ensures cell survival and tissue integrity upon osmotic stress. This study highlights the conservation of the stress response in algae and land plants and establishes Chlamydomonas as a unicellular plant model system to dissect the osmotic stress signaling pathway.

Maintaining cellular water homeostasis is vital in all living organisms, requiring various mechanisms to counteract differences in water availability between the intracellular and extracellular environment occurring during fluctuating environmental conditions ${ }^{1}$. Detailed mechanistic insight of the osmotic stress response has been established in unicellular model systems such as yeast, but knowledge is more limited in photosynthetic organisms ${ }^{2}$. We sought to advance our understanding by establishing the unicellular alga Chlamydomonas (Chlamydomonas reinhardtii) as a model system to study osmotic stress through a systems biology approach and identify functionally conserved components across the green lineage.

\section{Chlamydomonas transcriptomic and post-translational response to osmotic stress}

To establish a temporal map of the osmotic stress responses in Chlamydomonas, we performed a transcriptomic analysis of the wild-type strain CC-4533 across a 24-hour time course using two osmotic challenges, $\mathrm{NaCl}$ and mannitol, treatments that substantially reduce cell growth but are non-lethal (Extended Data Fig. 1a-b). We identified a total of 1,456 differentially-regulated genes $(\mathrm{FC}>2, \mathrm{FDR}<0.01)$, with a peak of regulation after 1 hour (Table S1, Table S2, Extended Data Fig. 1c-d). Up-regulated genes were enriched $($ FDR $<0.05)$ in annotations associated with 
genes were associated with the cell cycle, rRNA metabolic processes, chloroplast fission, and deoxyribonucleotide metabolism (Fig. 1a). Consistent with these data, we found that an osmotic challenge induced an increase in the number of vesicles and starch puncta in Chlamydomonas cells (Fig. 1b, Extended Data Fig. 2a-b), using fluorescent reporters that mark these compartments ${ }^{3}$. These regulatory patterns suggest that osmotic stress induces a pause in general cellular functions and activates stress response genes, a pattern also observed in yeast and land plants upon osmotic stress $^{4,5}$. The Chlamydomonas genome includes homologous gene families found in both Saccharomyces (Saccharomyces cerevisiae, fungi), and Arabidopsis (Arabidopsis thaliana, land plant $)^{6}$. Chlamydomonas osmotic stress-responsive genes have homologs in both Arabidopsis and Saccharomyces, although the likelihood of differential expression upon osmotic stress is greater in Arabidopsis homologs compared to Saccharomyces homologs (24\% and 6\% respectively) (Material and methods, Extended data Fig. 3, Table S2). Our analysis therefore suggests that, although the Chlamydomonas transcriptional response to osmotic stress shares features with both Arabidopsis and Saccharomyces, it is more similar to Arabidopsis.

While the abscisic acid (ABA) hormone signaling pathway controls much of the transcriptional response to osmotic stress in Arabidopsis, in Chlamydomonas, we did not find enrichment of gene ontologies associated with either ABA regulated genes or related cis-elements (eg. ABA RESPONSE ELEMENT or G-box) (Extended Data Fig. 1e-f) ${ }^{7,8}$. This, in conjunction with the lack of orthologous genes to ABA receptors, or other downstream components, in the Chlamydomonas genome ${ }^{6}$, suggests the absence of a functional ABA-mediated transcriptional response to osmotic stress in this species. A feature of Chlamydomonas cells that is shared with animals and certain plant lineages is the formation of motile cilia. We found an enrichment of genes encoding ciliary proteins being differentially expressed in Chlamydomonas under osmotic stress $^{9}$ (Fisher's Exact Test=1E-05) (Extended Data Fig. 2c, Table S2), as well as a substantial reduction in flagellar length upon osmotic stress (Fig. 1b). Together, these data demonstrate that osmotic stress induces rapid changes in Chlamydomonas cellular organization that are likely under the control of signaling pathways distinct from the dominant ABA-dependent pathway of land plants.

To uncover early signaling components of the Chlamydomonas osmotic stress response pathway, we adapted untargeted high-resolution mass spectrometry in conjunction with metabolic 
identified 33 Chlamydomonas differentially phosphorylated proteins $(\mathrm{FC}>2, \mathrm{FDR}<0.01)$ encompassing a variety of functions including: transcriptional regulation (MYB and bZIP family proteins) and flagellar machinery (Flagella-associated proteins (FAP), FAP 69 and FAP 88), among others. We found several proteins with homologs that when mutated in Arabidopsis have osmotic sensitive phenotypes, such as; Ubiquitin protease UBIQUITIN-SPECIFIC PROTEASE $16(\mathrm{UBP} 16)^{10}$ and ENHANCED EM LEVEL (EEL), a bZIP transcription factor homologous to ABA-INSENSITIVE 5 (ABI5) $)^{11,12}$. Similarly, we identified several proteins with Saccharomyces homologs previously shown to be involved in osmotic stress, including; ribosomal stalk protein RIBONUCLEASE P (RPP1A) ${ }^{13}$, VESICLE-ASSOCIATED MEMBRANE PROTEINASSOCIATED PROTEIN (SCS2) ${ }^{14}$, and INOSITOL POLYPHOSPHATE 5-PHOSPHATASE (IP 5-P), a Phosphoinositol binding protein involved in cytoskeletal reorganization ${ }^{15}$. Among the Saccharomyces orthologous proteins, 8 out of 14 were shown to be phosphorylated upon osmotic stress, while only 3 out of 16 showed phosphorylation in Arabidopsis (Fig. 1c, Table S4). We identified 56 proteins with orthologs that are also phosphorylated in Arabidopsis upon osmotic stress with lower confidence (FC >1.5, FDR <0.01) (Extended Data Fig. 4, Table S4). Our dataset includes 11 unannotated proteins which we renamed PHOSPHORYLATED UPON OSMOTIC STRESS IN CHLAMYDOMONAS (POC).

Altogether our transcriptional and phosphoproteomic analysis established the immediate targets of osmotic stress signaling in Chlamydomonas and highlights components most likely to be conserved across the green lineage and with non-photosynthetic eukaryotes.

\section{Discovery of novel genes with roles in osmotic stress}

Microbial model systems provide advantages for genome-wide, forward genetic screens due to the ease of culturing thousands of genotypes simultaneously. We utilized a recently developed barcoded genome-wide mutant library ${ }^{16}$ and subjected Chlamydomonas cells to various osmotic stresses. We used four different perturbations: $\mathrm{NaCl}$, mannitol, Polyethylene glycol

114 (PEG), and hypo-osmotic stresses and identified 76 genes with a growth defect when mutated across at least one stress: 27 for $\mathrm{NaCl}, 34$ for mannitol, 13 for hypoosmotic, 2 for $\mathrm{PEG}$ (FDR $<0.3$; Fig. 2a-c, Table S5-6). While several replicates were performed for each condition, few significant

117 mutants were identified across replicates, suggesting that the screens had not been performed to saturation. We validated our mutant phenotypes by performing secondary screens of 140 mutants 
selected according to different statistical criteria (Table S7, Extended Data Fig. 5), validating 55\% of the hits. We identified genes encoding: 9 transporters, 4 flagella-associated proteins, 7 kinases, 2 phosphatases, 11 nuclear localized proteins, among others. From this list of high confidence hits we identified 34 and 53 Saccharomyces and Arabidopsis orthologs, respectively (FDR $<0.3$, Table S5). Interestingly, 24 genes did not have any previous annotation in Chlamydomonas, Arabidopsis, or Saccharomyces, and therefore we renamed them OSMOTIC GROWTH DEFECTIVE IN CHLAMYDOMONAS (OSMO). Among our top hits, we identified HYPEROSMOLALITYINDUCED Ca INCREASE 1 (CreOSCA1) and MscS-like (CreMSL) that mediate the initial events of osmotic stress signaling in Arabidopsis ${ }^{17,18}$. Both showed sensitivity to $\mathrm{NaCl}$ and mannitol, acclimation to osmotic stress and flagella defects, and cellular localization comparable to the Arabidopsis homolog (Extended Data Fig. 6) ${ }^{19,20}$. Below, we describe several examples dissecting our functional genomics results by cellular functions.

We found that core osmotic stress signaling components such as kinases: CALCIUMDEPENDENT PROTEIN KINASE (CPDK) and MITOGEN-ACTIVATED PROTEIN KINASE KINASE KINASE (MAPKKK) homologous to Cre03.g153150 and Cre13.g576600, and phosphatases: PROTEIN PHOSPHATASE 2C (PP2C) and SHEWENELLA-LIKE PROTEIN PHOSPHATASE (SLP), homologous to Cre03.g211073 and Cre03.g185200, have conserved functions between kingdoms, playing central roles in Arabidopsis and Saccharomyces osmoregulatory pathways ${ }^{1,2}$ (Fig. 2c, Table S6) . Our screens have osmolyte specificity as shown by the number of membrane transporters necessary for Chlamydomonas growth under $\mathrm{NaCl}$, but not other osmotic stresses, including SODIUM/HYDROGEN EXCHANGER (NHX, Cre01.g034150) and ENDOPLASMIC RETICULUM-TYPE CALCIUM-TRANSPORTING

142 in sodium detoxification in plants and fungi ${ }^{21,22}$ and other transporters such as a SULFATE

143 TRANSPORTER (SULTR, Cre10.g457750) and a PHOSPHATE TRANSPORTER (PHT, 144 Cre09.g402775).

$145 \quad$ Nuclear localized factors identified in sensitive mutants included: transcription factors with 146 homology to the APETALLA-2 family (AP2, Cre19.g750947 and Cre08.g385350) and 147 SQUAMOSA PROMOTER PROTEIN-LIKE (SPL, Cre03.g185200); a WD-40 repeat-containing 148 protein, Cre10.g465650, previously linked to drought stress in Arabidopsis ${ }^{23}$; and a core 
Cre16.g682950), among others. Interestingly we found two genes whose Arabidopsis homologs were previously shown to function in acclimation to osmotic stress: a STARCH BRANCHING ENZYME (SBE, Cre10.g444700) and ABA DEFICIENT 3 (ABA3, Cre03.g152000) a gene encoding a molybdenum cofactor sulfurase that has been demonstrated to participate in both ABA and ABA-independent stress pathways within Arabidopsis ${ }^{24,25}$. Not surprisingly, we also found limited overlap in the gene lists identified through transcriptomics and functional genomics, with just five genes exhibiting a significant growth defect and transcriptional response upon osmotic stress; an AP2 transcription factor, SBE, a motor microtubule protein KINESIN 7 (KIN7, Cre13.g568450), a proprotein convertase subtilisin (Cre05.g242100) and Cre17.g735650, which is not annotated. Overall, our functional genomics identified new high confidence genes involved in osmotic stress signaling and corroborates low overlap between transcriptomics and functional genomics, previously reported for other model systems, such as yeast ${ }^{26,27}$.

Our systems biology approach points to the involvement of multiple organelles in the osmotic response pathway. We picked genes with two validated mutant alleles from our secondary screens and studied the localization of their encoded proteins fused to VENUS (Fig. 2d, Extended Data Fig. 7). We were able to localize four proteins to various cellular organelles, including a Potassium channel (KCN11, Cre06.g278111) at the contractile vacuole, S-palmitotransferase (PAT, Cre06.g277000) at the Golgi apparatus, LETHAL EMBRYONIC OSMOTIC (LEO, Cre12.g524950) at the chloroplast, and PROFILIN (PRO, Cre10.g427250) in the cytoplasm and at the nuclear envelope. Together our multi-omics characterization of Chlamydomonas points to a conservation of many osmotic signaling pathway components between algae, fungi, and land plants and identifies new components with high confidence for future studies.

\section{Novel osmotic stress pathways conserved across the green lineage}

Previous studies have found that using a combination of model systems is advantageous

175 for functionally characterizing conserved gene functions, such as using yeast and mouse to dissect 176 the cell cycle, or Chlamydomonas and Arabidopsis to uncover the photosynthetic apparatus ${ }^{28}$. 177 Thus, to identify new osmotic cellular pathways conserved across the green lineage, we grew 178 Arabidopsis seedlings with mutations in genes homologous to Chlamydomonas osmo-sensitive 179 genes identified in our screens and analyzed the ability of their roots to acclimate to osmotic stress 
exhibiting osmotic phenotypes in both Chlamydomonas and Arabidopsis; including genes encoding for: MITOGEN ACTIVATED PROTEIN KINASE KINASE (MAPKK), GATED OUTWARDLY-RECTIFYING POTASSIUM CHANNEL

(GORK),

SPALMITOTRANSFERASE (PAT), PROFILIN 5 (PRF-5), and LETHAL EMBRYONIC OSMOTIC 1 (LEO1), a gene that encodes an uncharacterized putative GTP-binding protein (Fig. 3a, Extended data Fig. 5d).

Time-lapse imaging of the Arabidopsis primary roots with an introgressed plasma membrane marker, $L T I 6 b-Y F P^{29}$, revealed different patterns of root growth cessation upon osmotic stress and the cellular basis for these growth defects in the mutants (Fig. 3b-f). Under standard conditions, all mutant primary roots grew similar to wild-type except gork-1, which exhibited a loss of root hair anisotropic growth, producing round hair cells. Inflated root hairs were suppressed when the mutants were grown on either media lacking supplemented potassium, at low temperatures, or by removing sucrose from the media, suggesting that the abnormal growth and loss of cellular integrity in this mutant is caused by a growth-dependent intracellular accumulation of potassium (Extended data Fig. 8).

Wild-type plants transferred to $140 \mathrm{mM} \mathrm{NaCl}$ showed cell death in the late elongation and early differentiation zones, approximately $400-600 \mu \mathrm{m}$ from the root tip within 4-8 hours after transferring to stress. In comparison, treatment with $300 \mathrm{mM}$ mannitol did not affect cell viability. In contrast, we could score different spatiotemporal cell-viability phenotypes in our mutants (Fig. 3c-f). The mapkk-1 mutant showed cell death in the elongation-differentiation zone upon $\mathrm{NaCl}$ or mannitol treatment, but with a peak of damage to the root occurring at different times posttreatment (7-14 hours for $\mathrm{NaCl}, 7-10$ hours for mannitol). Pat-1 mutants showed cell death in the meristematic zone, 0-200 $\mu \mathrm{m}$ from the root tip, and elongation zone with a peak of cell death occurring 9 hours after transfer to stress treatment, which resulted in roots without epidermal cells within the first $400 \mu \mathrm{m}$ from the root tip. Similarly, mannitol treatment induced cell death to a minor degree in pat-1 mutants, starting 4 hours after transfer. Early cell death occurred in the meristematic and elongation zones of prf-5 mutants upon transfer to $\mathrm{NaCl}$, leaving most epidermal cells dead. Comparably, mannitol treatment promoted cell death at the meristematic-elongation zone boundary (a.k.a. transition zone) as early as 2 hours after treatment in prf-5 mutants.

210 Continuous cell death in the transition zone occurred in gork- 1 mutants after $\mathrm{NaCl}$ treatment, while 211 mannitol promoted cell death in the early elongation zone. Finally, cell death occurred in the leo- 
2121 mutant at late timepoints upon $\mathrm{NaCl}$ treatment, while no cell death happened upon mannitol

213 treatment. Together our phenotypic characterization indicates that all mutants exhibit sensitivity

214 to both $\mathrm{NaCl}$ and mannitol-mediated osmotic stress, though greater sensitivity was generally

215 observed with $\mathrm{NaCl}$. Mutants exhibited distinct patterns of cell viability defects, which suggests

216 different cellular mechanisms may be mediating the sensitivity to osmotic stress.

\section{The actin cytoskeleton reorganizes upon osmotic stress}

The Arabidopsis prf-5 mutant exhibited one of the earliest and most severe defects in cell viability amongst our characterized mutants, with most meristematic and elongating cells dying within the first 5 hours after transfer to stress. Profilin is an actin interacting protein that determines the dynamics of the actin cytoskeleton by controlling the rate of polymerization, bundling, and cable formation $^{30,31}$. We therefore hypothesized that osmotic stress may alter actin cytoskeleton dynamics and that the prf-5 mutant exhibits hypersensitivity to stress due to the inability to properly control this reorganization. To test this hypothesis we monitored actin dynamics in roots using the actin-binding domain of fimbrin fluorescent reporter, ABD2-GFP reporter ${ }^{32}$. Upon transfer to osmotic stress, both $140 \mathrm{mM} \mathrm{NaCl}$ and $300 \mathrm{mM}$ mannitol, root cells in the elongation zone underwent massive actin reorganization (Fig. 4a). An increase in the asymmetric distribution of actin filaments, skewness, revealed an increase in actin bundling together with a decrease in the filament number. Concomitantly, actin filament angle switched from being nearly parallel to the cell longitudinal axis of the cell to being perpendicular ${ }^{33}$ (Fig. 4a-b). Interestingly, visualization of actin dynamics in Chlamydomonas using Lifeact-NeonGreen, showed a similar response to osmotic stress with an increase in skewness (Extended data Fig. 10). Together these data suggest that actin reorganization upon osmotic stress is conserved across the green lineage.

Treatment of prf-5 mutants with $140 \mathrm{mM} \mathrm{NaCl}$ led to cell death in the elongation zone (Fig. $3 \mathrm{c}$, e). Treatments with lower concentrations of $\mathrm{NaCl}, 50 \mathrm{mM}$, promoted actin reorganization in the prf-5 mutant while no changes were observed in wild-type plants, suggesting that the prf-5

238 actin network is hypersensitive to osmotic stress (Fig. 4c-d). To localize PRF5 we generated a PRF5 fluorescent tag reporter driven by its own promoter. ProPRF5::PRF5-YFP is expressed in root hair cells of the elongation zone and differentiation zone as well as the last two layers of the

241 lateral root cap. Time-course imaging of the expression of PRF5 upon osmotic stress revealed the 242 downregulation of its expression during the initial hours after treatment (Extended data Fig. 10). 
243 Since our mutant analysis suggests that PRF5 inhibits NaCl-induced bundling, we hypothesize that

244 PFR5's normal function may be to delay the induction of osmotic-stress mediated actin bundling.

245 Calcium signaling is an important downstream mediator of abiotic stress ${ }^{34,35}$. To test whether the

246 defects observed in prf-5 are due to a lack of a stress response or a hyper induction of a stress

247 response, we monitored calcium dynamics in roots transferred to a mild $\mathrm{NaCl}$ concentration in

248 wild-type and prf-5 backgrounds (Fig. 4e-f). Wild-type plants transferred to $50 \mathrm{mM} \mathrm{NaCl}$ showed

249 infrequent calcium spikes. In contrast, $p r f-5$ mutants showed ectopic calcium spikes within the

250 elongation region and an increase in spiking frequency immediately after transfer to stress. Thus,

251 osmotic-stress mediated calcium dynamics are hyperactive in prf5 mutants, supporting the

252 hypothesis that the dynamical properties of the actin cytoskeleton tune cellular acclimation and

253 sensitivity to changes in water availability.

\section{Discussion}

Our work provides a framework for comparing the conservation and diversification of osmotic stress tolerance pathways. Across Chlamydomonas, yeast and Arabidopsis we have observed common mechanisms to reduce investment in growth and to redirect resources towards osmotic homeostasis. Interestingly, divergence between species occurs frequently in the types of organelles where acclimatory mechanisms function and the downstream signaling pathways that coordinate the response. While Chlamydomonas utilizes flagella as a primary mechanism of exploring the environment, root growth would be the analogous process in seed plants such as Arabidopsis, and both are suppressed under osmotic stress. These data suggest that organisms evolve tolerance mechanisms that suit their specific ecological niche and the physiological processes that they normally carry out.

The actin cytoskeleton is a dynamic network controlling diverse cellular activities such as 267 vesicle trafficking and ion channel activity ${ }^{36}$. Early cellular responses to osmotic stress involve 268 shifts in the balance between endocytosis and exocytosis of proteins important for growth, such as 269 cellulose synthase complexes ${ }^{37-39}$, and ion homeostasis, such as ion channels that regulate cell 270 turgor pressure ${ }^{18}$ and calcium signaling ${ }^{19}$. We show here that osmotic stress causes a dramatic 271 sequestration of actin into bundles and cables, which may effectively inhibit many of the cellular

272 processes that require filamentous actin. This large scale change in actin organization may provide 273 structural support to the cell or may be part of a quiescence program. Further work is needed to 
274 explore the signaling and biophysical implications of this broadly conserved stage of osmotic275 stress acclimation.

\section{Acknowledgments}

We thank Xiobo Li for sharing early versions of the mutant library; Silvia Ramundo for generous gift of pRAM118 plasmid; Masayuki Onishi for generously sharing Lifeact-NeonGreen Chlamydomonas strain. Heather Cartwright and the Carnegie imaging facility for microscopy support. Members of the Dinneny lab for helpful discussions. We thank Gregory A Barret-Wilt, and the phosphoproteomic core facility for help and assistance in the generation of the Chlamydomonas osmotic phosphoproteome. We thank Christopher J. Staiger for providing ABD2-GFP lines and Wolf Frommer for providing GcaMP6 reporter.

This project was supported by grants awarded to J.R.D from the NIH NIGMS (R01 GM12325901) and a Faculty Scholars grant from the Simons Foundation and Howard Hughes Medical Institute (55108515); grants awarded to M.C.J. from NIH (DP2-GM-119137), NSF (MCB1146621 and MCB-1914989) and the Simons Foundation and HHMI (55108535); grants awarded to M.R.S IOS PGRP No. 2010789 and MCB No. 9143816; Simons Foundation fellowships of the Life Sciences Research Foundation awarded to R.E.J. and J.V.B.; and an EMBO long term fellowship (ALTF 1450-2014) awarded to J.V.B.

\section{Author contributions}

R.E.J., T.X., and J.V.B. analyzed RNA-seq data: BM and MS performed phosphoproteomic experiments; R.E.J., F.F., and J.V.B. prepared mutant pools, performed treatments, and processed all samples, and analyzed data with guidance from in M.C.J.; T.V generated Chlamydomonas fluorescently tagged lines and validated mutant insertion sites; T.V., K.K., and J.V.B. performed and analyzed Chlamydomonas secondary screenings; L.W. generated Cre10.g45568 reporter line; K.K. provided technical assistance and help with the analysis of the phosphoproteomic dataset; M.G. and J.O. performed initial screen of Arabidopsis orthologous phenotypes; J.V.B. performed all the other experiments; J.V.B. and J.D. design the experiments and analyze the data; J.V.B wrote the initial draft of the manuscript; J.D and J.V.B wrote the final version of the manuscript with input from all authors. 
Figures

a

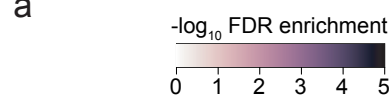

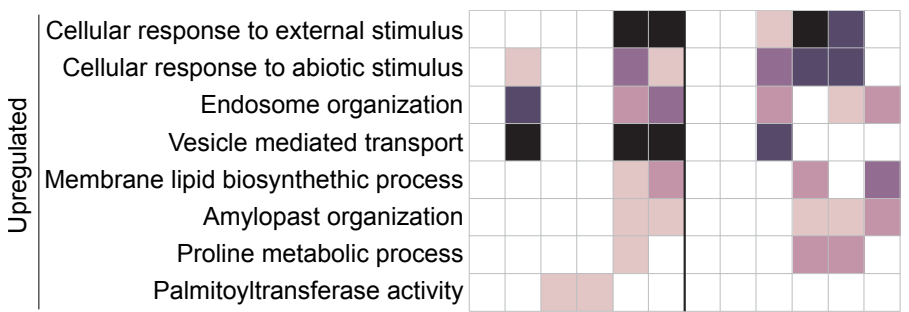

$15^{\prime} 30^{\prime} 60^{\prime} 4 \mathrm{~h} 6 \mathrm{~h} 24 \mathrm{~h} 15^{\prime} 30^{\prime} 60^{\prime}$ 4h $6 \mathrm{~h} 24 \mathrm{~h}$

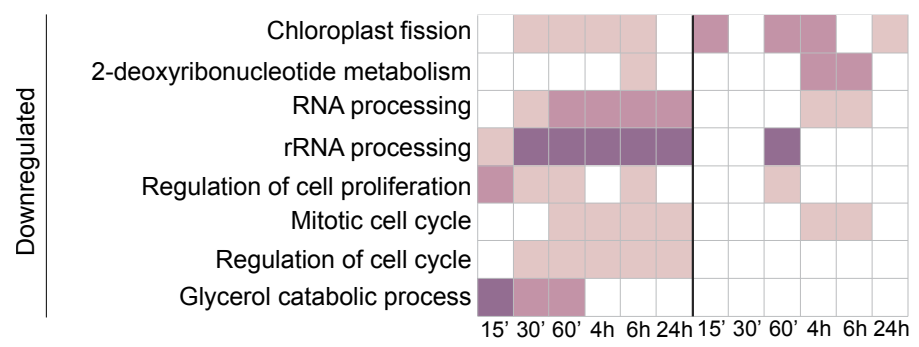

C

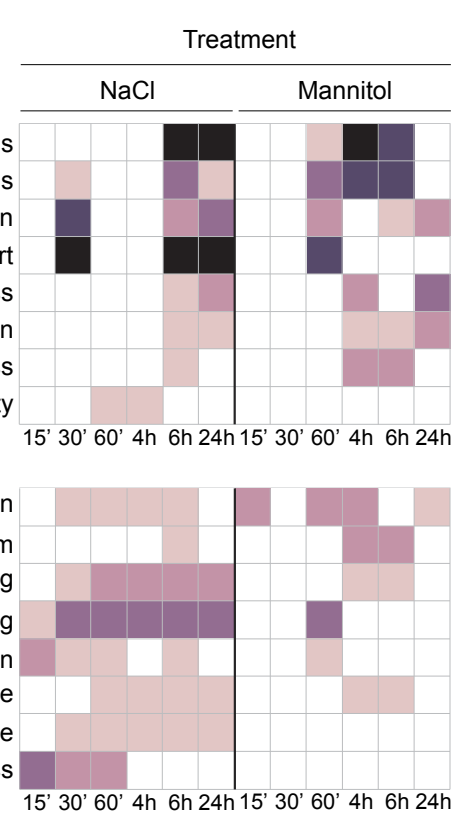

b
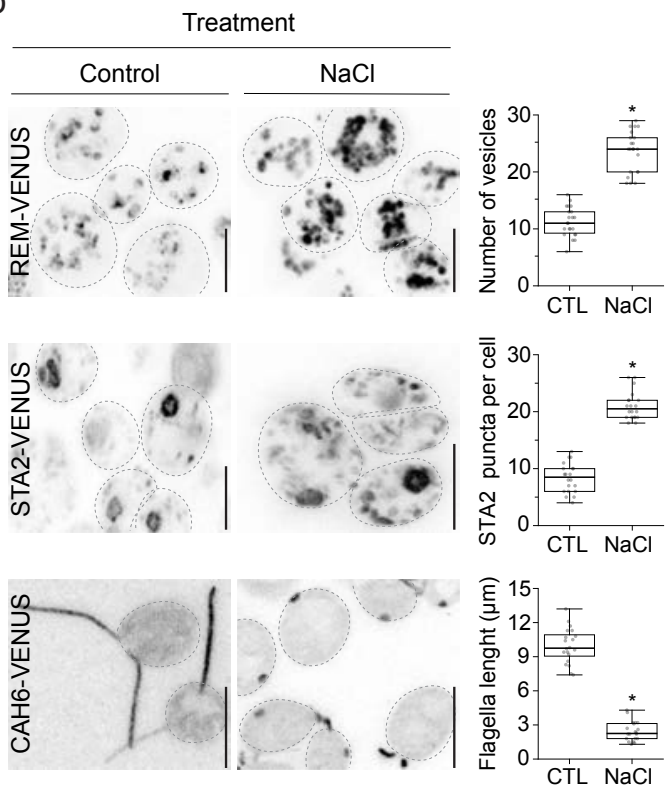

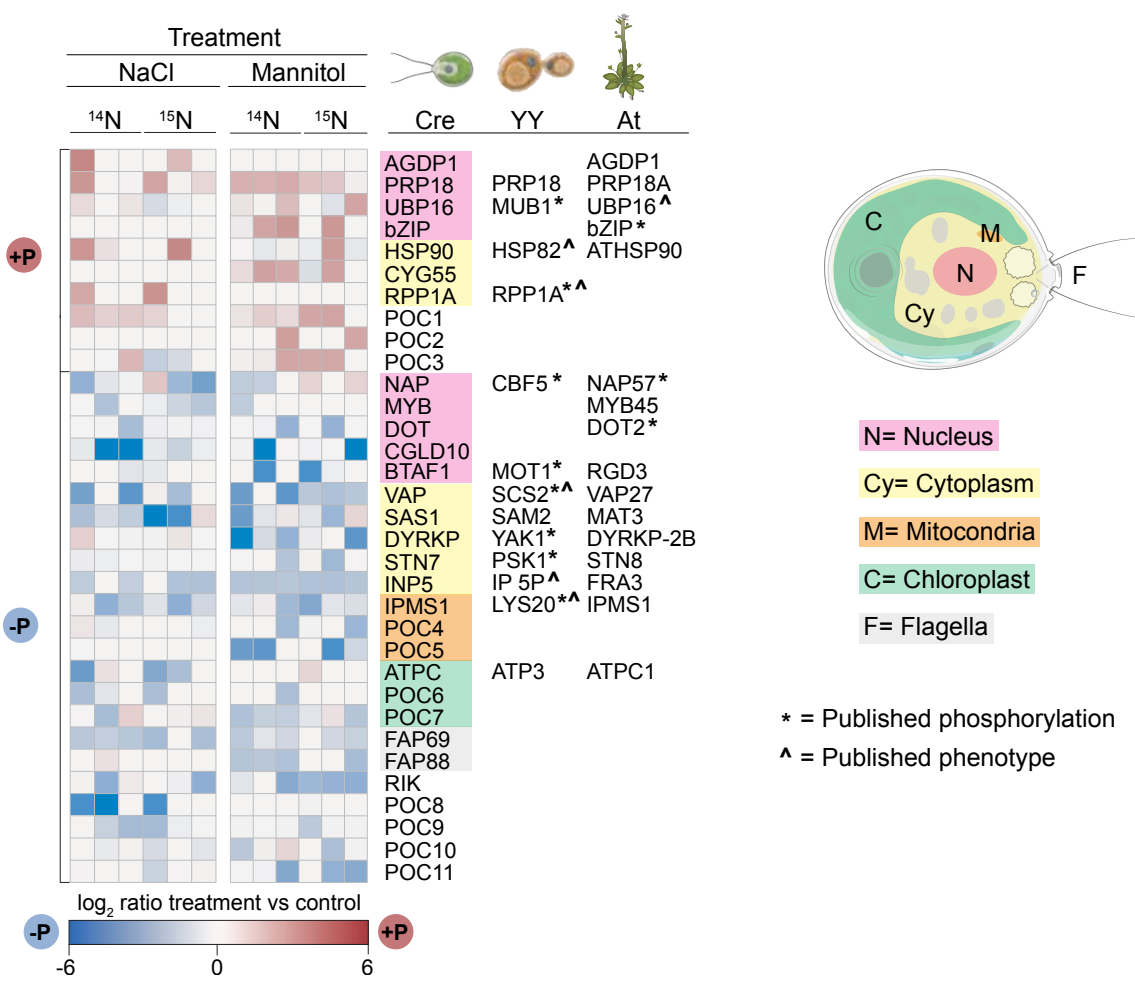

Figure 1. Chlamydomonas reinhardtii shared and divergent osmotic stress responses with other eukaryotes. a, $300 \mathrm{mM}$ mannitol at the indicated time points, ' and $\mathrm{h}$ indicate minutes and hours respectively (See Extended Data 13, Table S1, S2). b. Confocal images of fluorescently tagged Chlamydomonas cells with different fluorescent markers ${ }^{3}$ under control conditions and treatment with $100 \mathrm{mM} \mathrm{NaCl}$ for 30 hours. Cellular markers used: Endosomes, Cre16.g661700: REMORIN, labels the Golgi apparatus and secretory pathway (REM-VENUS); Starch, 
313 Cre17.g721500: STARCH SYNTHASE 2 (STA2), labels pyrenoid starch and starch granules (STA2-VENUS); 314 flagella, Cre12.g485050: CARBONIC ANHYDRASE 6 (CAH6) labels flagella (CAH6-VENUS). (Right) 315 Quantification of the number of vesicles in REMORIN cells, STA2 puncta and flagella length in control and cells 316 treated with $\mathrm{NaCl} . \mathrm{n}>20$ cells per condition. Scale bars $=10 \mu \mathrm{m}$. $\mathbf{c}$, Heat map representing proteins differentially 317 phosphorylated (FC $>2$, FDR $<0.01$ ) upon 5 min treatment with $100 \mathrm{mM} \mathrm{NaCl}$ or $300 \mathrm{mM}$ mannitol. Color code 318 represents protein localization according to PredAlgo ${ }^{40}$ or associated functions. ${ }^{*}$ Represents published 319 phosphorylated proteins upon osmotic stress, ^ represents proteins with a described role in osmotic pathways (See 320 Table S2, S3 and Extended Data 3). 
a

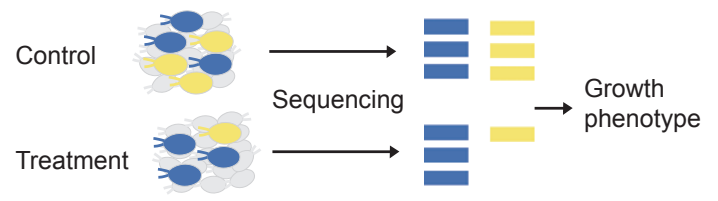

b
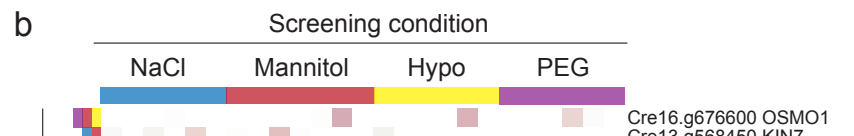

$\pi$
$\frac{\pi}{2}$

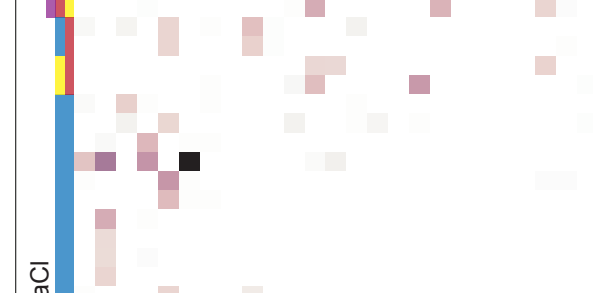

re16.9676600 OSMO re13.9568450 KIN7
Cre12.9554250 LPB1

Cre02.9147500 OSMO2

Cre12.9516950 SAS6

Cre06.9256700 OSMO3

Cre05.9242100 SDD

Cre01.g034150 NHX

Cre09.g402775 PHT
Cre07.g325752 FAP

Cre03.g149800 MAP

Cre04.g214050 MOT

Cre13.9576600 MAPKKK

Cre09.9396850 OSMO5

Cre13.g577300 GLOX

Cre03.g153150 CDPK

Cre10.g433900 HUWE1

re12.g5514350 OSMO

Cre06.9263950 ECA

Cre19.9750947 AP2 2 (A)

Cre08.9385350 AP2

Cre09.9410200 OSMO

Cre11.g477850 TSN

Cre16.g667150 OSMO8

Cree7.9318551 OSMO9

Cre11.g481250 OSMO10

Cre12.g523050 CCT11

Cre15.g641200 OAC

Cre02.g111550 HT1

Cre16.9691754 OSMO11

Cre12.9560550 PDE

Cre10.g428600 OSMO12

Cre10.g433750 PAP1

Cre17.g745547 FIP

Cre02.g143151 SBP

Cre03.g2 200600 KIFC

Cre12.9550900 NCP1

Cre03.g152000 ABA3

Cre17.9735650 OSMO13

Cre12.9516300 OSMO14

Cre09.9393950 FTSH

Cre16.9651400 OSMO15

Cre03.g086400 OSMO16

Cre12.9500500 STM3

Cre12.9514700 PPH

Cre10.9430900 SBP

Cre13.9571520 OSMO18

Cre16.9682950 RAD9

Cre08.9380400 MAD

Cre11.g477150 SMC
Cre01.g003650 OSMO19

re01.g003650 OSMO

Cre06.9428450 SDP

Cre12.9524950 LEO

Cre01.g054250 MAX

Cre06.g282250 OSMO20

Cre03.g185200 SLP

Cre04.g220850 ABC

Cre10.g444700 SBE

Cre01.g012200 SPL

Cre06.g271150 FAP

Cre16.9658950 OSMO2

Cre14.g619800 SPO

Cre17.9715801 OSMO22

Cre07.9337300 DYRKP

Cre04.g219700 BBS9

Cre07.9344600 EDA

Cre14.92516950 OSMO23

Cre06.9261200 SBH

岗

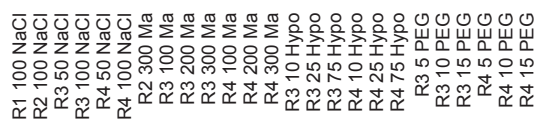

$-\log _{10}$ FDR
C

$\begin{array}{lll}\text { Screen } & \text { Orthology } & \text { Phenotype } \\ \square \mathrm{NaCl} & \text { Cre-At-Sc } & * * \text { At-Sc } \\ \square \text { Mannitol } & \text { Cre-At } & * \text { Sc } \\ \text { PEG } & \text { Cre } & * \text { At } \\ \text { Hypoosmotic } & \end{array}$

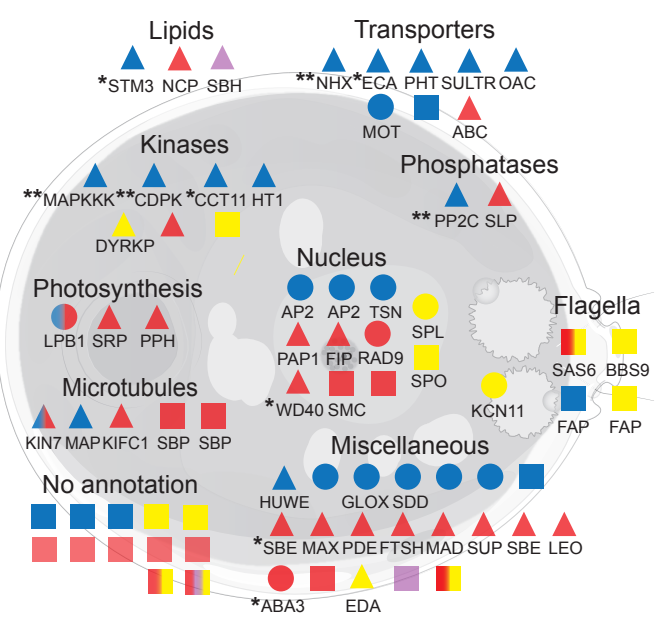

d
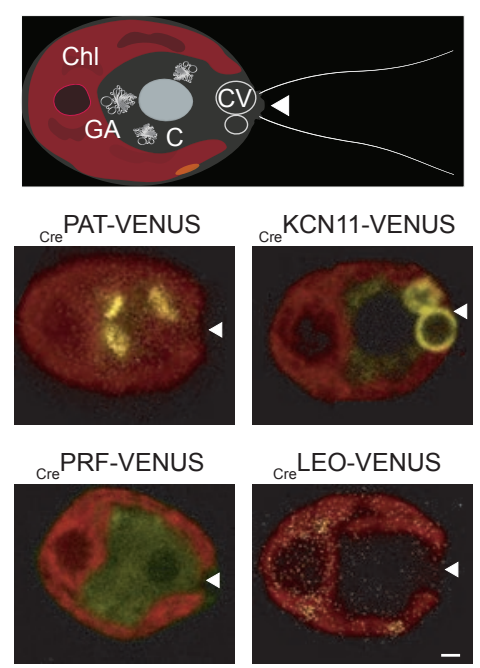

Figure 2. Chlamydomonas osmotic stress pathways involve different organelles. a, Diagrammatic representation of barcode mutant screens. Unique barcodes allow genome-wide screening of Chlamydomonas mutants in a pool. Mutants sensitive to osmotic stress can be identified because their barcodes will be less abundant after growth under treatment ( $\mathrm{NaCl}$, mannitol, $\mathrm{PEG}$, hypoosmotic) compared to control conditions. b, Heat map representing significance 
328 genome-wide barcoded screens upon different osmotic stresses (FDR $<0.3$ ). Hit orthologs to S. cerevisiae (Sc) or A. 329 thaliana (At) based on reciprocal BLAST $(<1 \mathrm{E}-10)$. Osmotic phenotype represents hits with a previously described 330 role in osmotic stress in S. cerevisiae (Sc.), A. thaliana (At) or both (See Table S5). d, Confocal images of 331 Chlamydomonas cells showing the subcellular localization of proteins fused to VENUS fluorescent protein. Cartoon 332 represents different organelles in Chlamydomonas: $\mathrm{Chl}=$ chloroplast, $\mathrm{GA}=$ Golgi apparatus, $\mathrm{C}=$ cytoplasm, $\mathrm{CV}=$ 333 contractile vacuole. Red color shows chloroplast autofluorescence and yellow shows VENUS fluorescence. Scale bar $334=1 \mu \mathrm{m}$. 
a

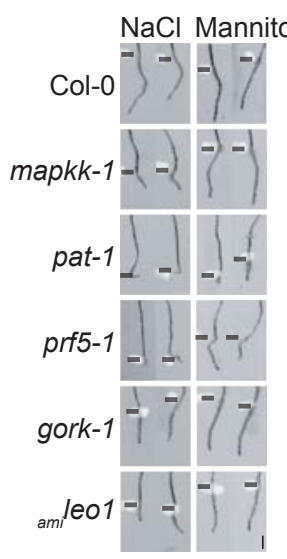

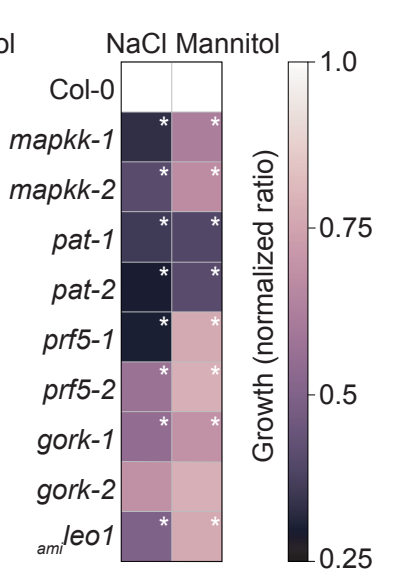

C
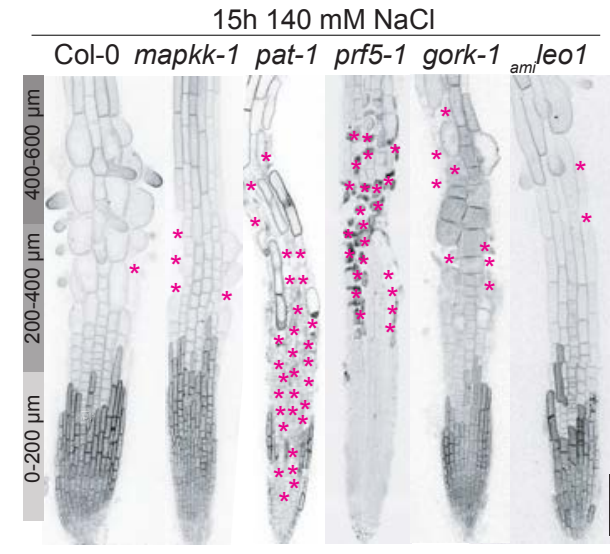

e

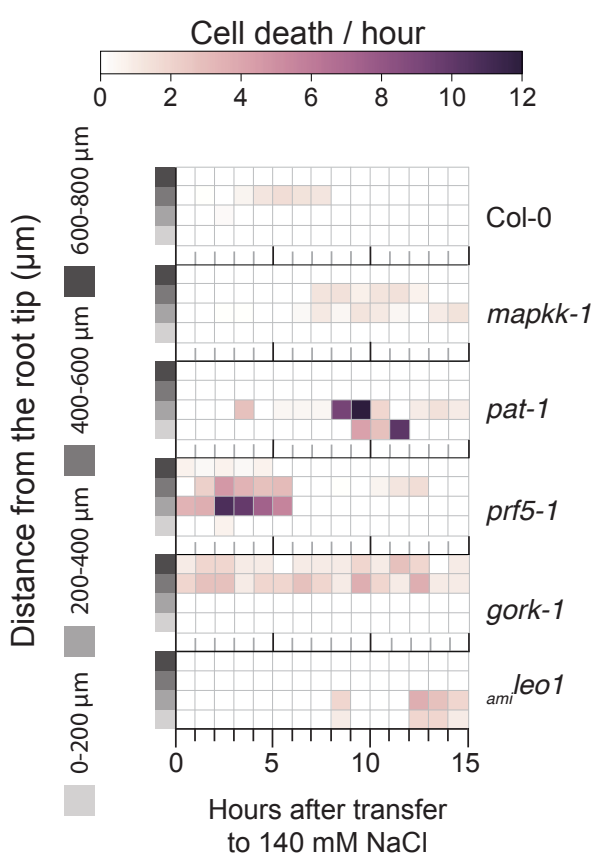

b

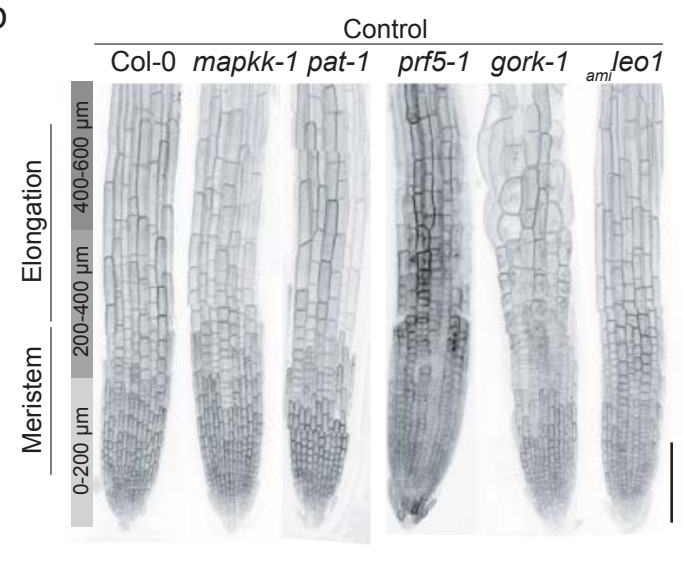

d

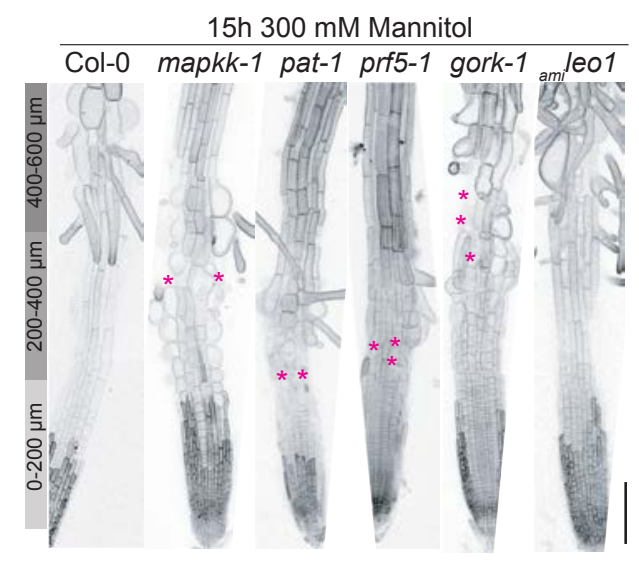

f

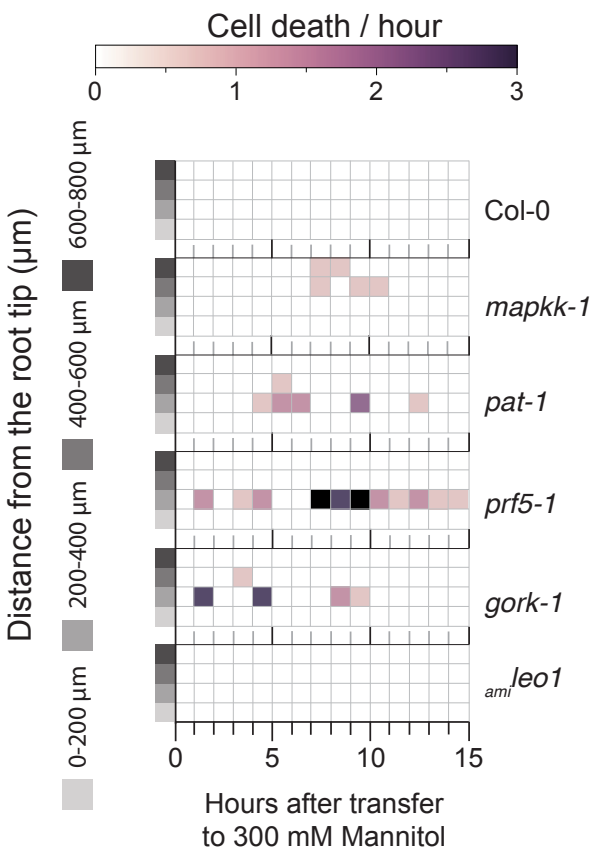


$340 \mathrm{mM}$ mannitol, root growth ratio upon transfer was normalized to the growth of wild type upon stress treatment. *

341 Indicates $p$-value $<0.05$, two-way ANOVA. $\mathbf{b}$, Confocal images of 4 days post germination primary roots, plasma 342 membrane marked by LTI6b-YFP fluorescence as in C and D. Scale bar $=100 \mu \mathrm{m}$. c, Confocal images of 4 days post 343 germination root tips, 15 hours after transfer to $140 \mathrm{mM} \mathrm{NaCl} .{ }^{*}$ Indicates cell death. Scale bar $=100 \mu \mathrm{m}$. d, Confocal 344 images of 4 days post germination root tips, 15 hours after transfer to $300 \mathrm{mM}$ mannitol. * Indicates cell death. Scale $345 \mathrm{bar}=100 \mu \mathrm{m}$. e, Quantification of root cell death after transfer to $140 \mathrm{mM} \mathrm{NaCl} . \mathrm{n}>10$ roots for each genotype. f, 346 Quantification of root cell death after transfer to $300 \mathrm{mM}$ mannitol. $\mathrm{n}>10$ roots for each genotype. 

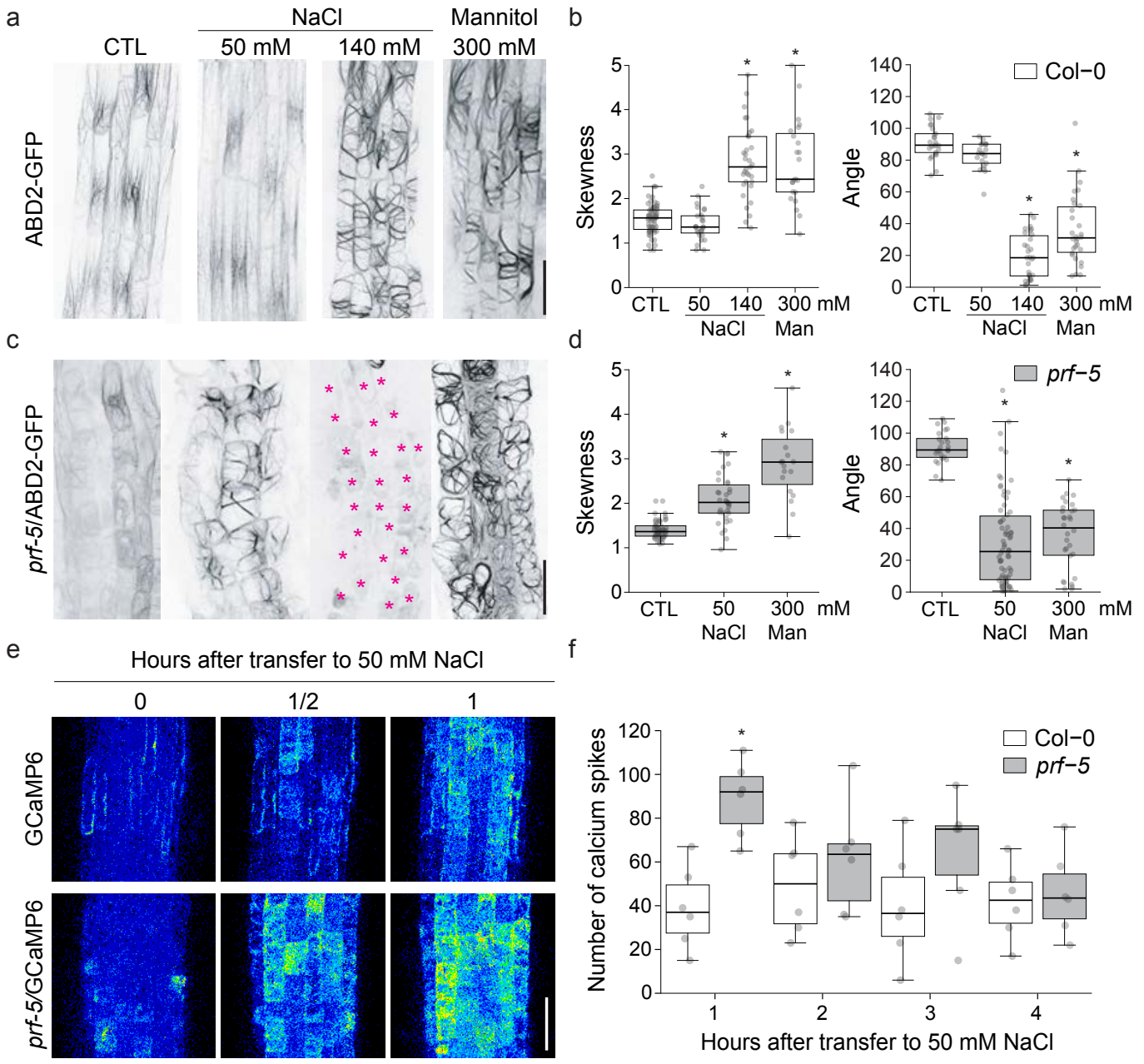

Figure 4. Osmotic stress promotes actin bundling in the root elongation zone. a, Actin localization in the Arabidopsis primary root elongation zone of wild-type roots on control conditions (CTL), transferred to $50 \mathrm{mM} \mathrm{NaCl}$, $140 \mathrm{mM} \mathrm{NaCl}$ or $300 \mathrm{mM}$ mannitol for 16 hours. Images from treatments correspond to cells actively elongating at the time of transfer. Actin filaments were visualized with the actin binding domain of the FIMBRIN protein tagged with green fluorescent protein (ABD2-GFP). Scale bar $=50 \mu \mathrm{m}$. b, Quantification of skewness and angle of actin filaments relative to the cell radial axis. Each point represents a cell that was actively elongating when roots were transferred. * Represents $p$-value $<0.05$, two-way ANOVA. c, Actin localization in the Arabidopsis root elongation zone of prf-5 roots on control conditions (CTL), transferred to $50 \mathrm{mM} \mathrm{NaCl}, 140 \mathrm{mM} \mathrm{NaCl}$, or $300 \mathrm{mM}$ mannitol for 16 hours. Images from treatments correspond to cells actively elongating at the time of transfer. Actin filaments visualized with ABD2-GFP. Scale bar $=50 \mu \mathrm{m}$. d, Quantification of skewness and angle of actin filaments relative to the cell radial axis. Each point represents a cell that was actively elongating when roots were transferred. * Represents $\mathrm{p}<0.05$, two-way ANOVA. e, 16 colors LUT confocal images of primary root elongation zone expressing GCaMP6, and $p r f-5 / G C a m p 6$. f, Hourly average number of $\left[\mathrm{Ca}^{2+}\right]$ spikes in wild-type and $p r f-5$ cells expressing GCaMP6 treated with $50 \mathrm{mM} \mathrm{NaCl}$. 
a

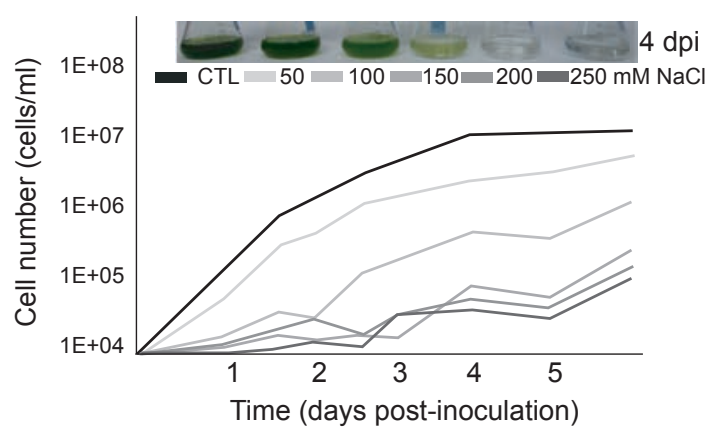

C

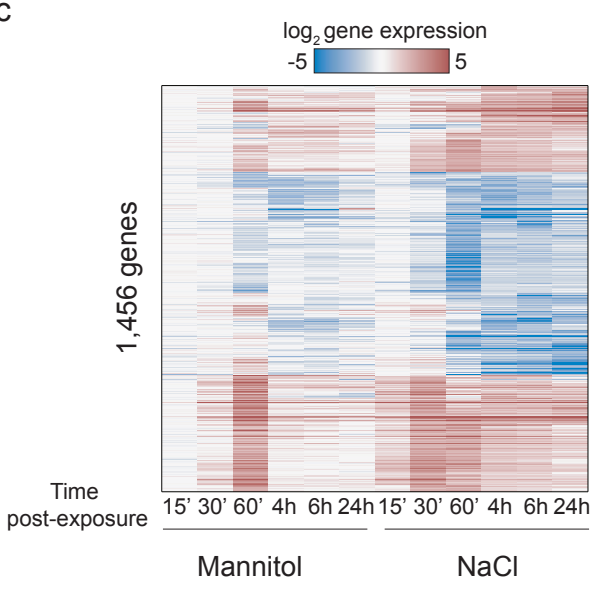

b

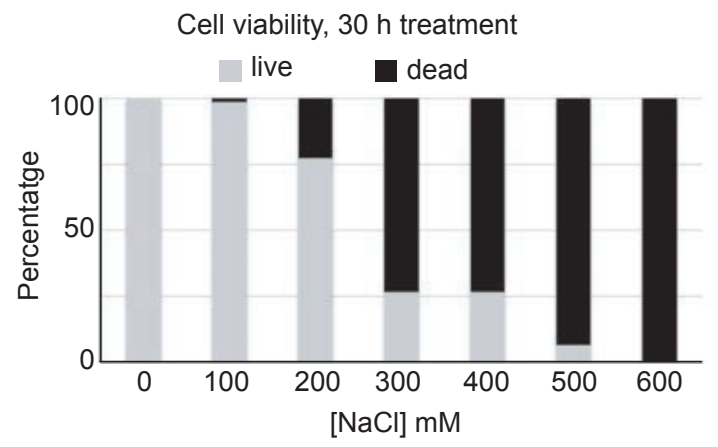

d

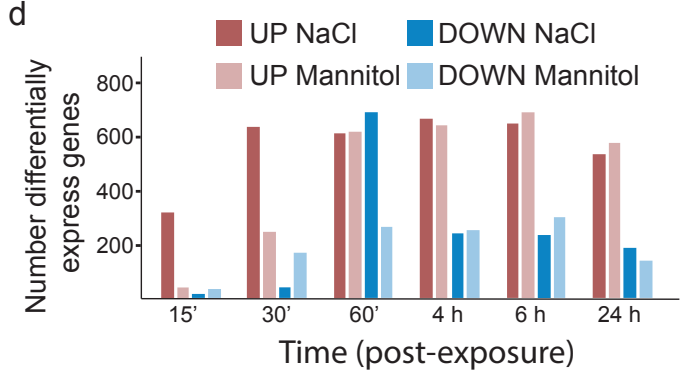

e

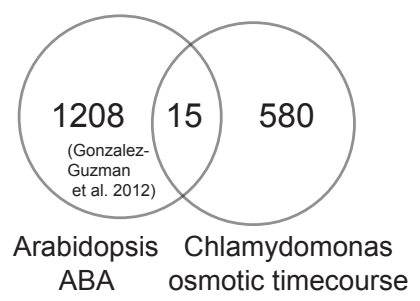

f \\ $-\log _{10}$ FDR enrichment \\ \begin{tabular}{llllll|}
\hline 0 & 1 & 2 & 1 & & \\
\hline & 1 & 2 & 3 & 4 & 5
\end{tabular} \\ Chlamydomonas Arabidopsis \\ Response to water deprivation \\ Response to water \\ Response to reactive oxygen species \\ Response to salicylic acid stimulus \\ Response to ethylene stimulus \\ Response to jasmonic acid stimulus \\ Response to salt stress \\ Response to abscisic acid stimulus \\ Response to osmotic stress \\ Transcription factor activity \\ Response to gibberellin stimulus \\ Response to heat \\ Trehalose biosynthethic process \\ Time post-exposure

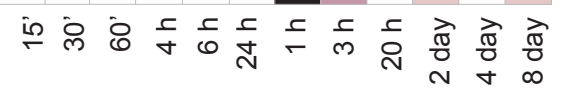 \\ $\mathrm{NaCl}$ \\ $\mathrm{NaCl}$ (Geng et al. 2013) \\ ABA hormonal regulation. a, Growth curves of Chlamydomonas wild-type cells (CC-4533) grown under different
}


369 concentrations of $\mathrm{NaCl}$. b, Cell viability of Chlamydomonas wild-type cells treated with different concentrations of $370 \mathrm{NaCl}$ for 30 hours. Evans blue staining was used to assess cell viability. c, Heat map representing clustered $\log _{2}$ values 371 of 1,456 differentially regulated genes ( $\mathrm{FC}>2$, FDR $<0.01$ ) under $100 \mathrm{mM} \mathrm{NaCl}$ and $300 \mathrm{mM}$ Mannitol treatment at 372 different timepoints. d, Number of differentially expressed genes (FC $>2$, FDR $<0.01$ ) at different timepoints. e, 373 Overlap of Chlamydomonas differentially expressed genes during $\mathrm{NaCl}$ and mannitol time course with $A$. thaliana 374 orthologous and differentially regulated genes upon ABA treatment in A. thaliana. (A. thaliana ABA regulated genes 375 from Guzman et al 2012). f, Gene ontology analysis of differentially regulated genes across the Chlamydomonas time 376 course treated with $100 \mathrm{mM} \mathrm{NaCl}$ and $\mathrm{NaCl}$ time course of Arabidopsis roots $^{8}$ (See Table S1-S3). 
a
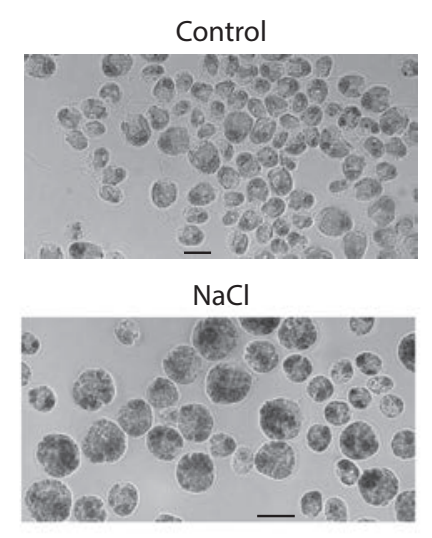

Lugol staining

b

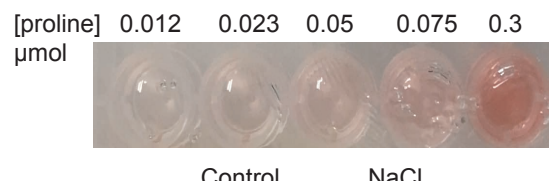

Control $\quad \mathrm{NaCl}$
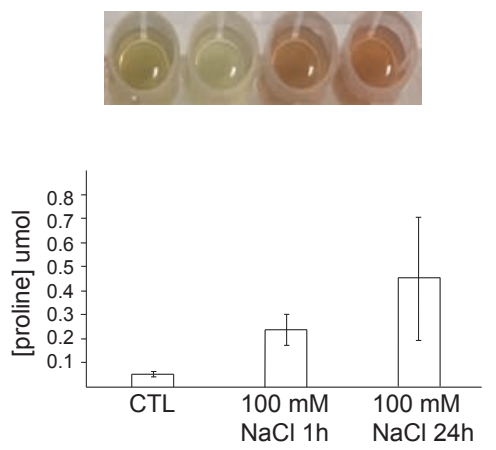

C

Expression of flagella associated genes

$\log _{2}$ gene expression

$-5 \square 5$ Gene ID
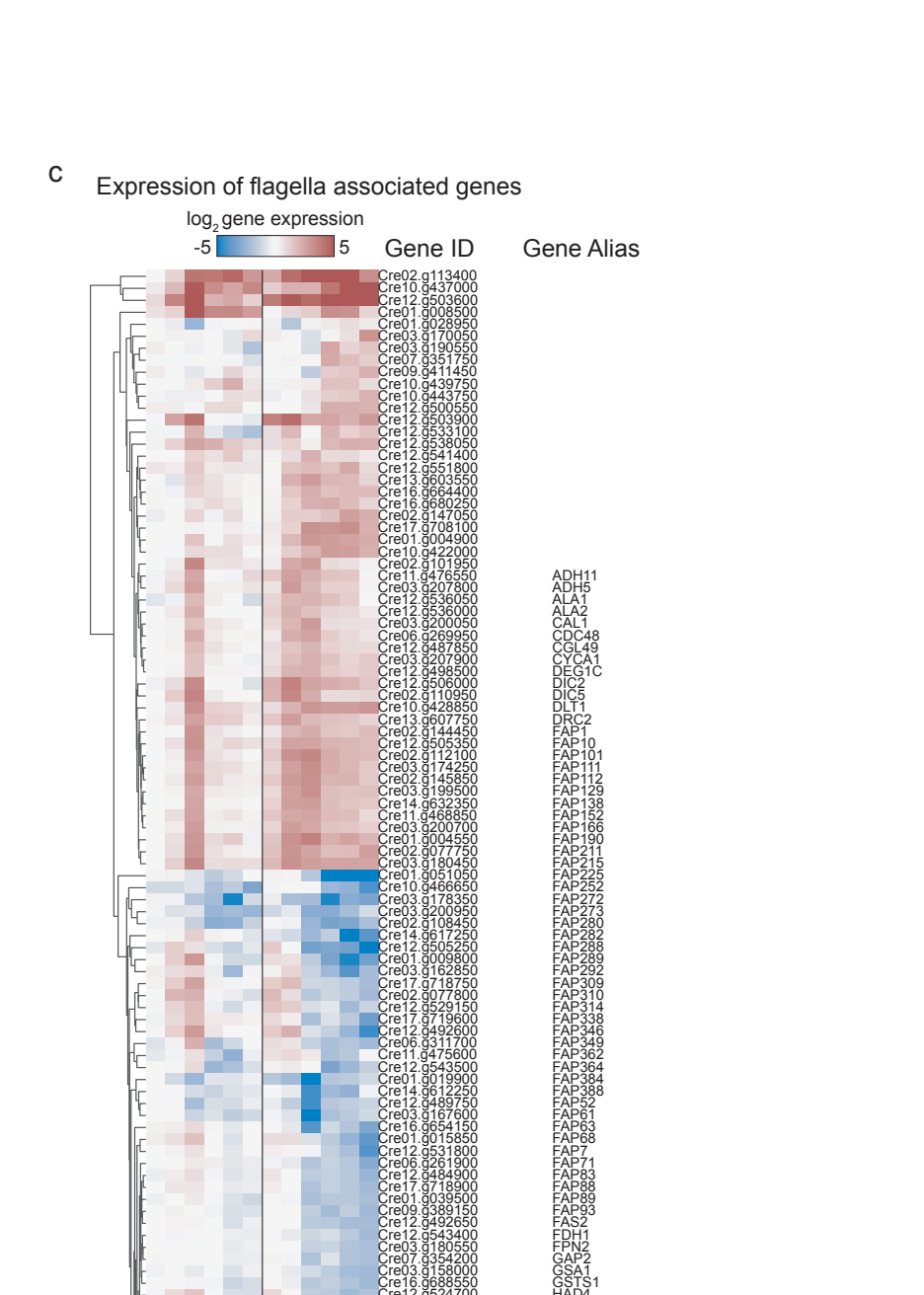

.

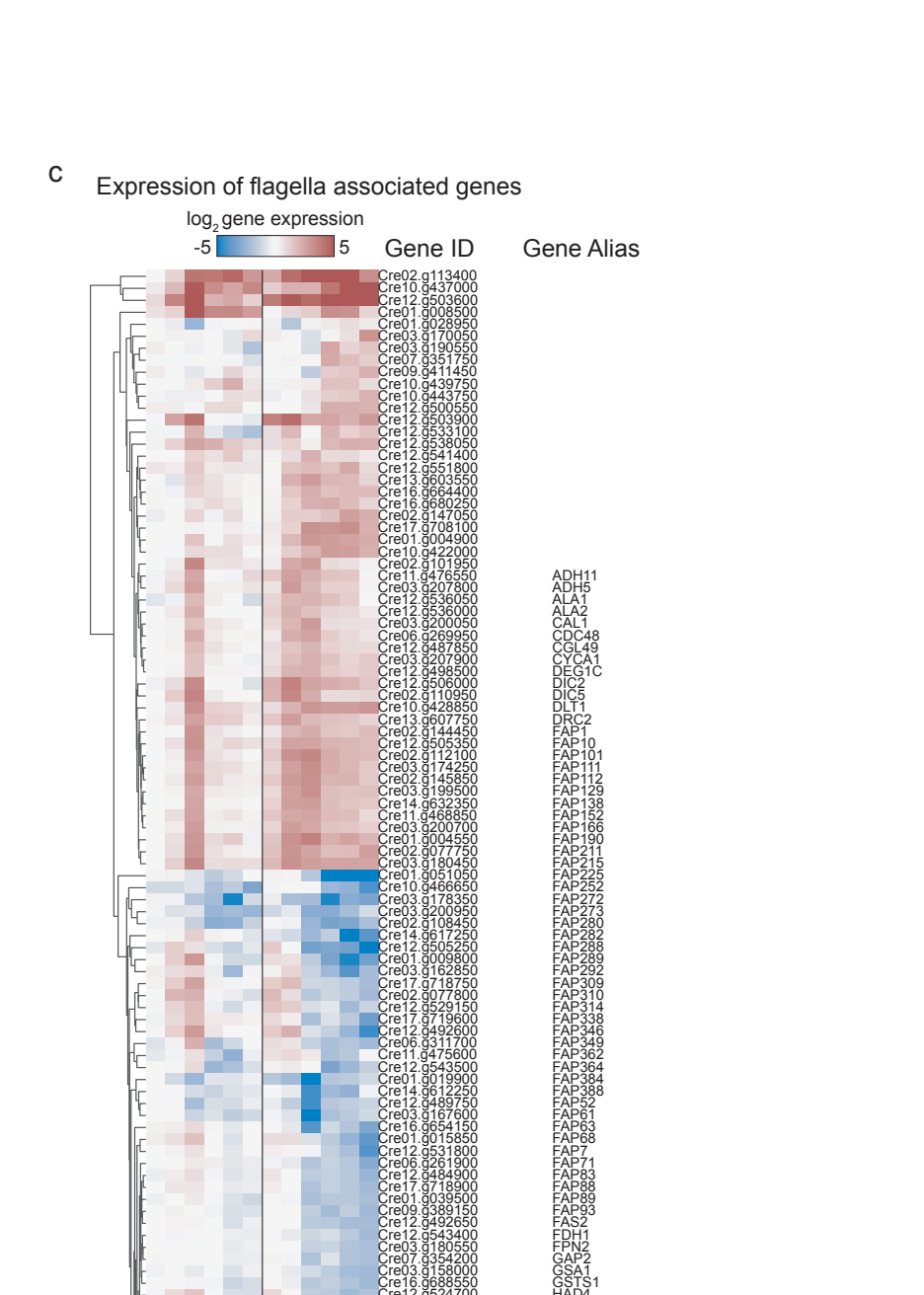

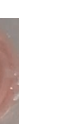

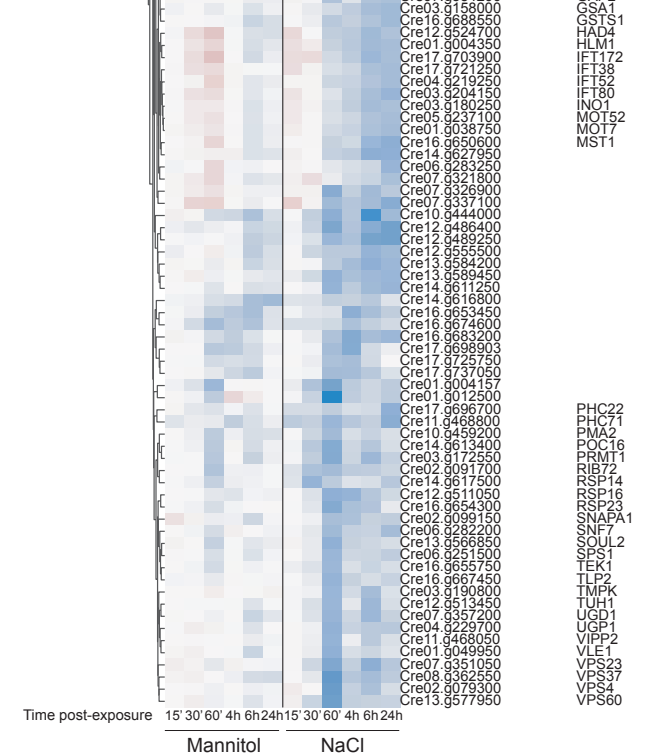

Extended Data 2. Chlamydomonas osmotic response affects flagella related genes and promotes starch and proline accumulation. a, Chlamydomonas cells stained with Lugol. (Top) CTL; Control Chlamydomonas cells in mid-early exponential phase stained with Lugol. (Bottom) $\mathrm{NaCl}$, Chlamydomonas cells in mid-early exponential phase treated with $100 \mathrm{mM} \mathrm{NaCl}$ for 20 hours and stained with Lugol. Scale bars $=10 \mu \mathrm{m}$. b. Proline quantification of Chlamydomonas cells upon $\mathrm{NaCl}$ treatment. c, Heat map representing $\log _{2}$ gene expression of Chlamydomonas Ciliary proteins (The Chlamydomonas Flagellar proteome project (http://chlamyfp.org/index.php)) differentially regulated in the Chlamydomonas osmotic transcriptome. (See Table S2). 


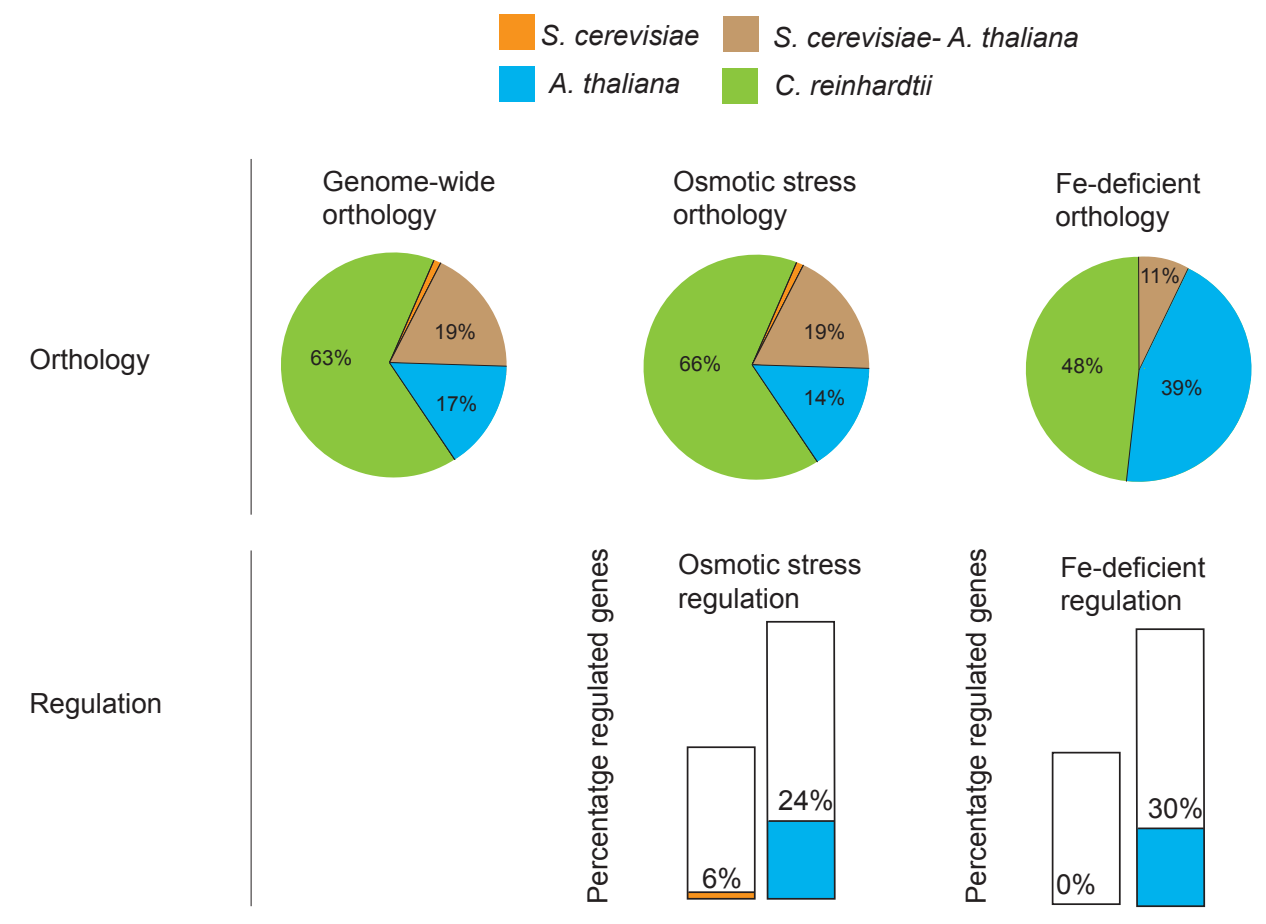

Extended Data 3. Conservation of the Chlamydomonas osmotic response across kingdoms. Pie charts representing the orthology of the Chlamydomonas genome with $S$. cerevisiae and A. thaliana genomes, orthology of differentially expressed genes in the Chlamydomonas osmotic time course and orthology of differentially expressed genes of Chlamydomonas iron-deficiency response; all othologies are based on reciprocal BLAST ( $>$ 1E-10) (See Material and Methods). Bars represent the percentage of genes differentially expressed upon osmotic stress or iron deficiency in Chlamydomonas with orthologous genes in S. cerevisiae (orange bars) or A. thaliana (blue) responding 


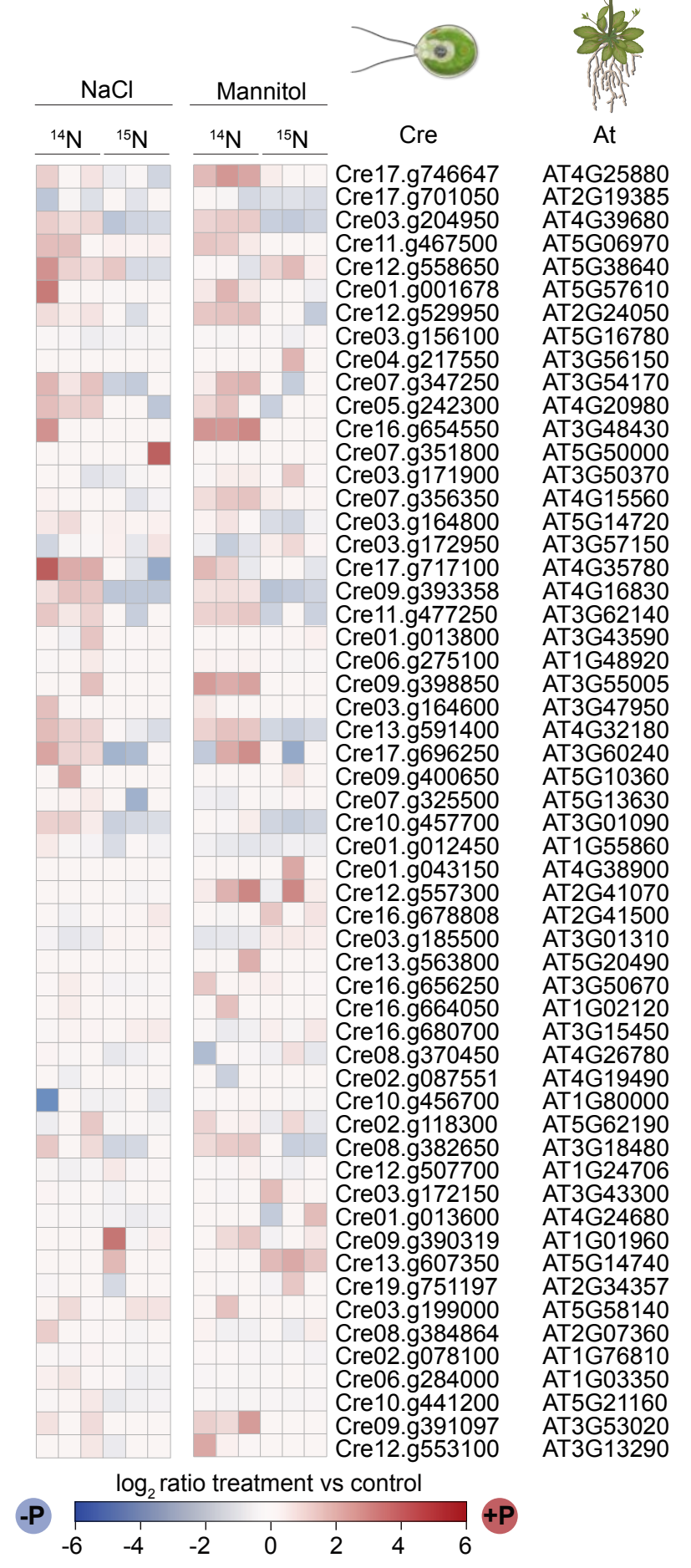

398 Extended Data 4. Phosphorylated proteins present in Chlamydomonas phosphoproteomic dataset and 399 osmotically phosphorylated orthologues in Arabidopsis. Chlamydomonas peptides differentially phosphorylated $400 \quad(\mathrm{FC}>1.5, \mathrm{FDR}<0.01)$ with $A$. thaliana orthologous that have previously shown to be differentially phosphorylated 401 upon osmotic conditions ${ }^{43,44}$ (See Table S3). 
a

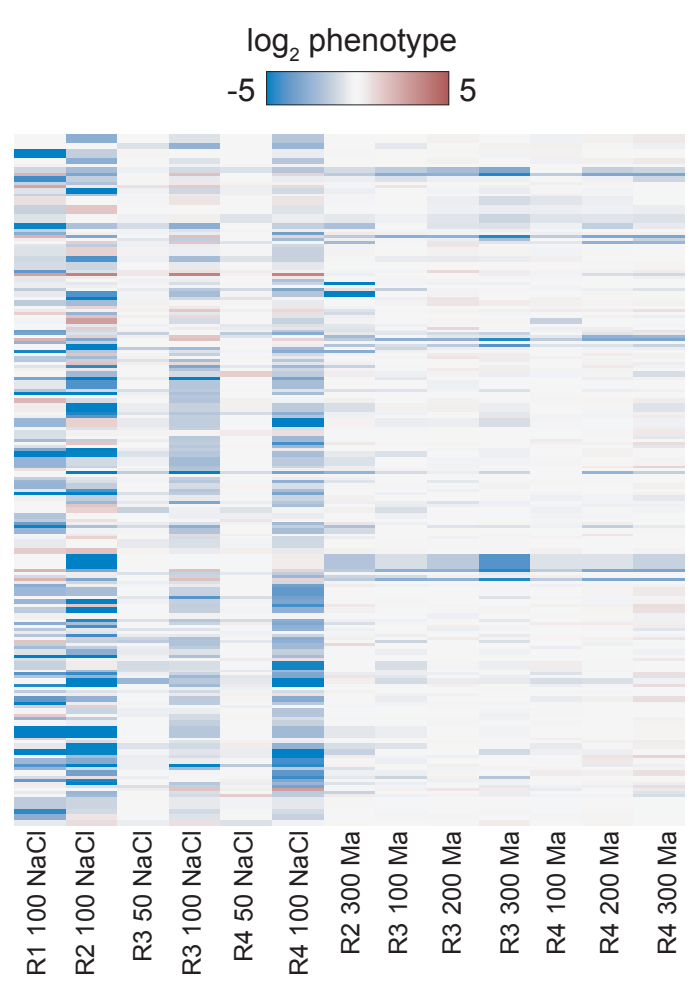

C

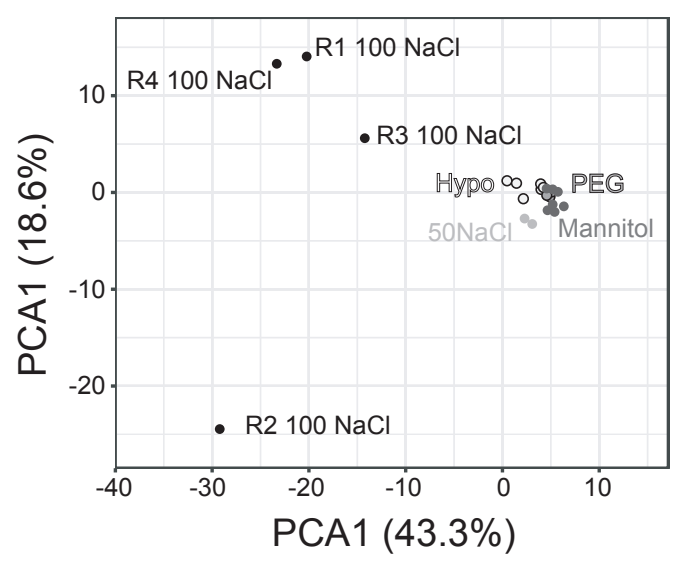

b

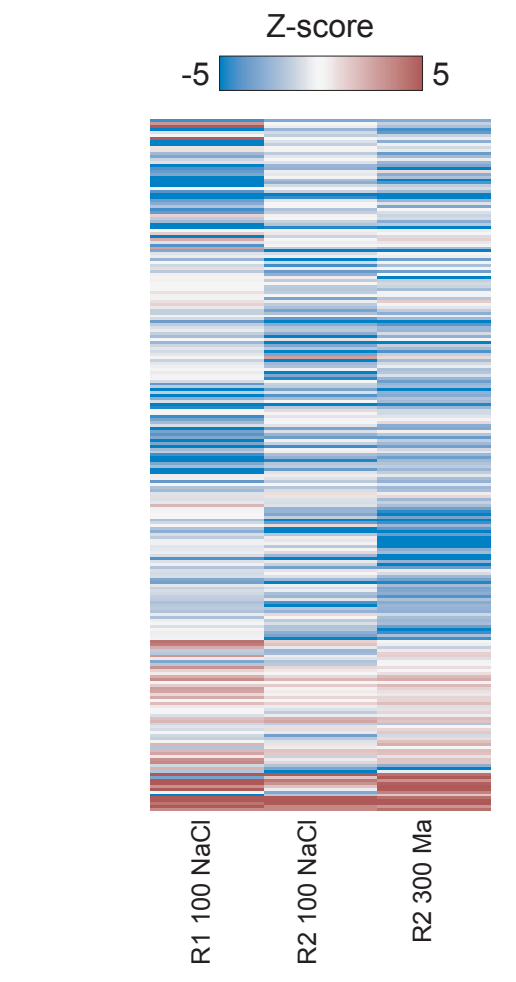

d

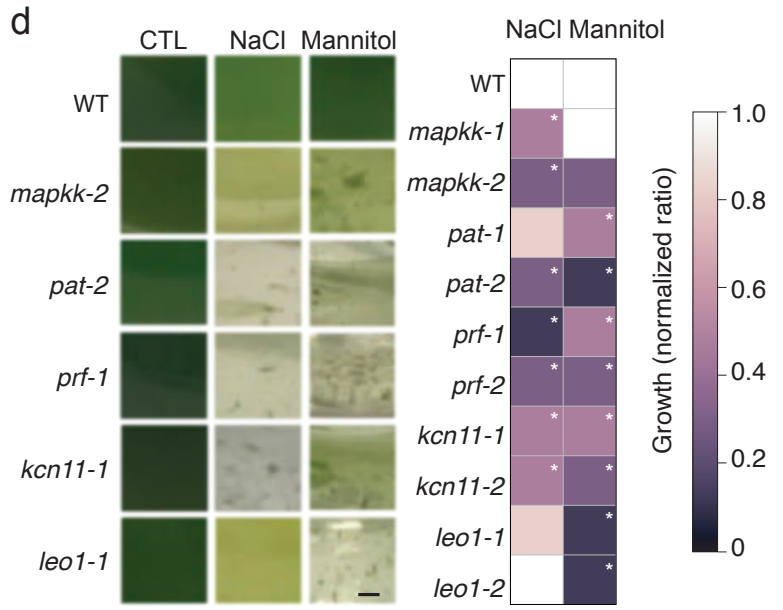

Extended Data 5. Validation of Chlamydomonas mutants with osmotic phenotypes. a, Median $\log _{2}$ phenotypes resulting from pooled mutant screens based on barcoded reads of hits selected for secondary screens (See Table S7). b, Z-score phenotypes of validated mutants. Z-score results from the quantification of chlorophyll RGB values (See Table S7). Note rows of mutants from A and B match horizontally. c, PCA of $\log _{2}$ median phenotype from different osmotic screens. d, Representative images of growth vessels 4 days post inoculation, grown in control media (CTL), $100 \mathrm{mM} \mathrm{NaCl}(\mathrm{NaCl})$ and $300 \mathrm{mM}$ mannitol (Mannitol). Scale bar $=1 \mathrm{~cm}$. (Right) Phenotype quantification 4 days post-inoculation under $100 \mathrm{mM} \mathrm{NaCl}(\mathrm{NaCl})$ and $300 \mathrm{mM}$ mannitol (Mannitol) of selected Chlamydomonas hits. Growth of mutant strains upon stress were normalized to growth of wild type upon stress. * Represents $p<0.05$ (twoway ANOVA). 
a

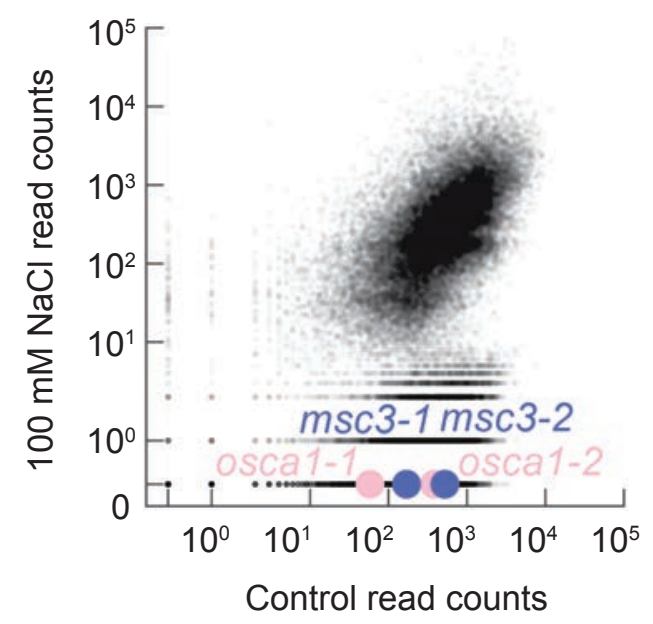

d

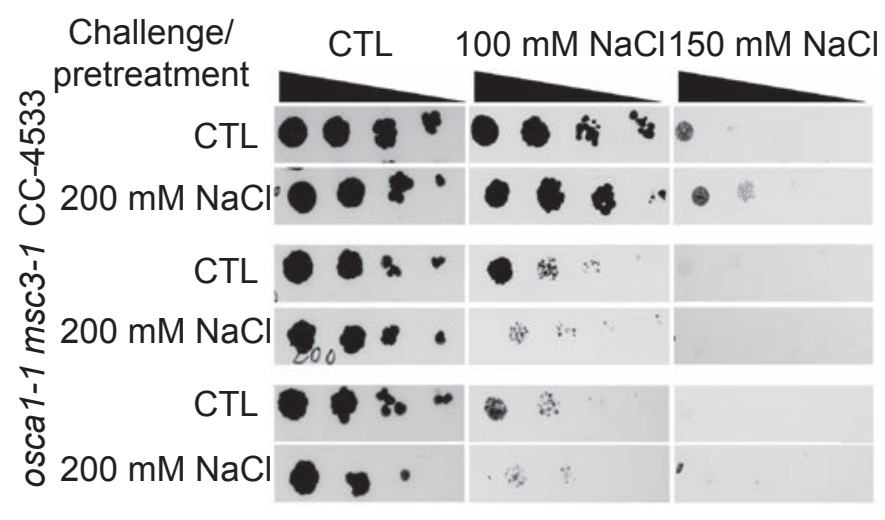

b

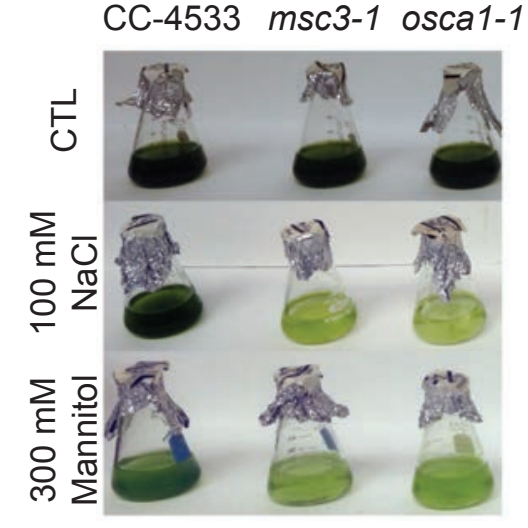

C

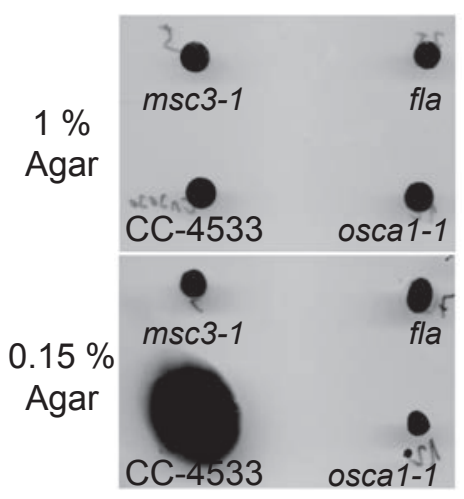

e

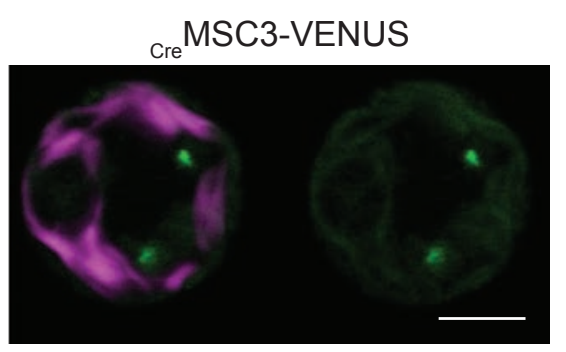

Extended Data 6. Functional conservation of osmoregulatory pathways across the green lineage. a, Scatter plot comparing barcode reads resulting from pooled screens in control condition and $100 \mathrm{mM} \mathrm{NaCl}$ treatment with osca and $m s l$ alleles highlighted. b, Growth of wild type (CC-4533) and msc3-1 and oscal-1 under control conditions (CTL), $100 \mathrm{mM} \mathrm{NaCl}$ and $300 \mathrm{mM}$ mannitol four days after inoculation. c, Flagella phenotypes of msc3-1 and oscal1 mutants. (Top) strains plated in $1 \%$ agar do not show colony differences, (bottom) strains plated in $0.15 \%$ agar show swimming differences based on colony size. As a positive control we used a flagella less mutant, fla. d, $m s c 3-1$ and oscal-1 mutants show acclimation defects to osmotic stress. Chlamydomonas CTL (CC-4533), msc3-1 and oscal-1 cells at the early mid-exponential phase $(2 \mathrm{E}+06 \mathrm{cell} / \mathrm{s} / \mathrm{ml})$ were plated at 10 -fold sequential dilutions (control raws, CTL) and Chlamydomonas cells at the exponential phase (2E+06 cells $/ \mathrm{ml})$ treated with $200 \mathrm{mM} \mathrm{NaCL}$ for 2 hours and plated in 10-fold dilutions ( $200 \mathrm{mM} \mathrm{NaCl})$. e, Confocal image of a Chlamydomonas cell expressing MSC 3 protein fluorescent signal to VENUS. 
a

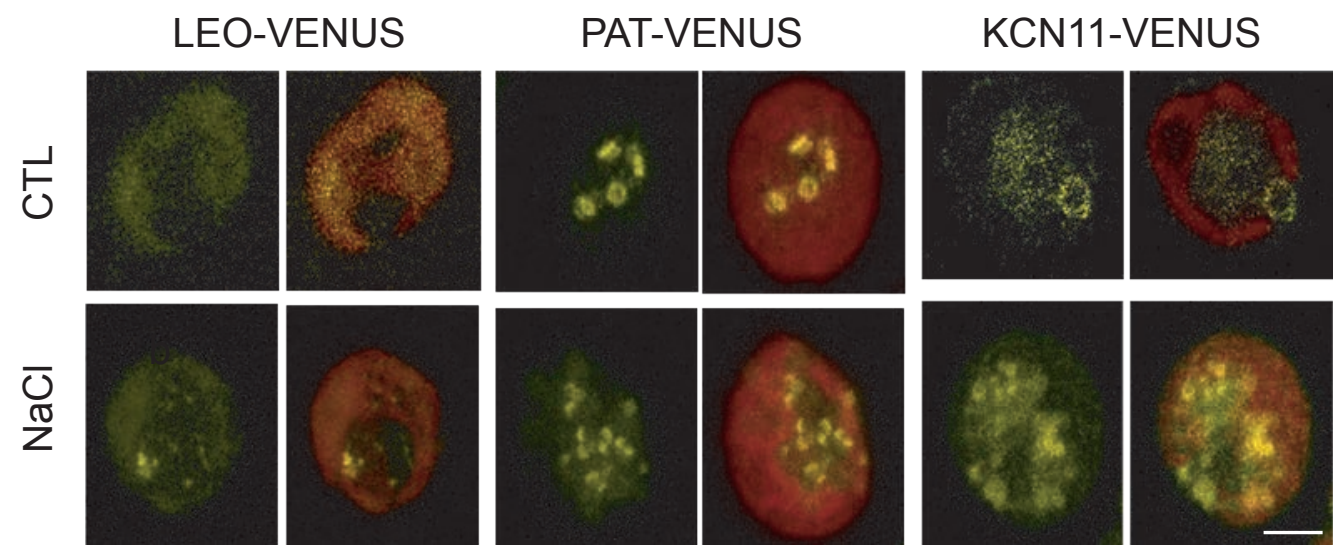

$\mathrm{b}$

Contractile Vacuole Marker

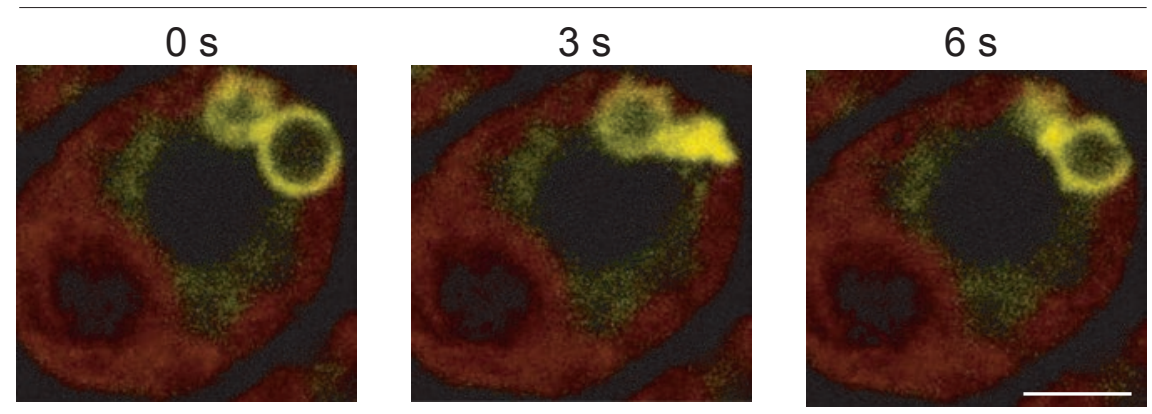

Extended Data 7. Protein localization upon osmotic stress of Chlamydomonas selected hits identified in the osmotic screens. a, Confocal images of Chlamydomonas cells expressing proteins fused to VENUS fluorescence protein under control conditions (CTL) and upon treatment with $100 \mathrm{mM} \mathrm{NaCl}(\mathrm{NaCl})$. Scale bar $=5 \mu \mathrm{m}$. b, Confocal images of Chlamydomonas cells expressing a contractile vacuole marker. Red color shows chloroplast 
a

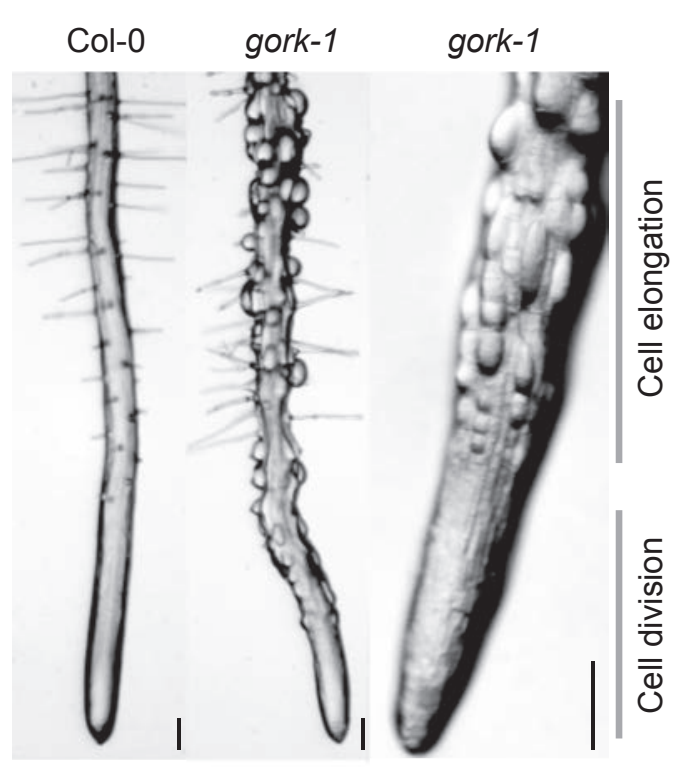

b

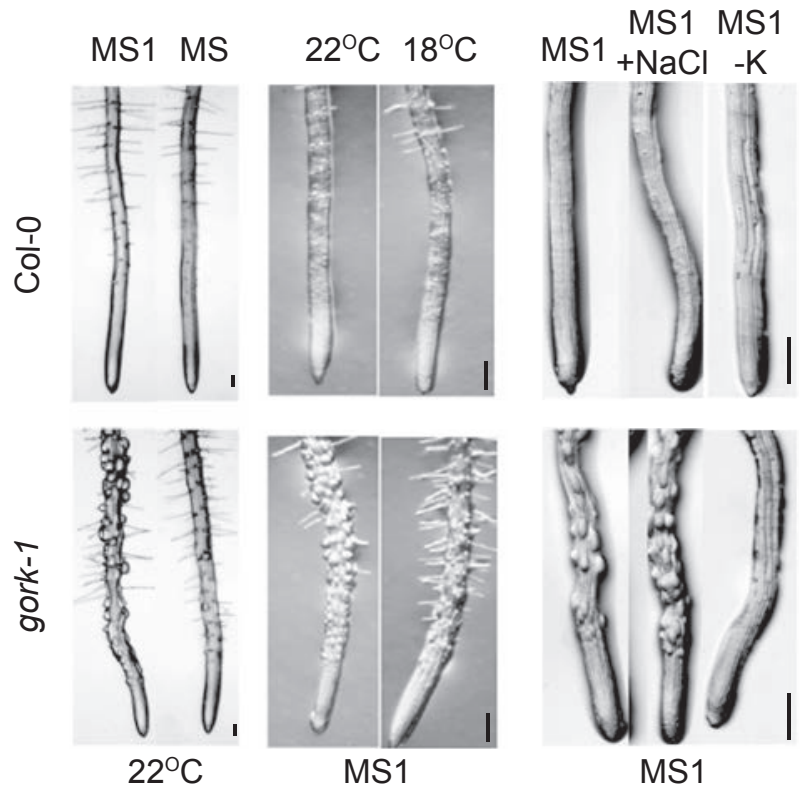

Extended Data 8. Growth dependent accumulation of potassium generates root hair inflated cells. a, Primary roots of 6 days-post germination wild-type (Col-0) and gork- 1 mutants. Scale bar $=100 \mu \mathrm{m}$. b, Primary root of 6 dayspost germination Col-0 and gork-1 mutants under different temperatures and sugar concentrations. MS1 media contains $1 \%$ sucrose, while MS does not contain sucrose. Scale bar $=100 \mu \mathrm{m}$. c, Primary root of 6 days-post germination Col- 0 and gork- 1 mutants $\mathrm{NaCl}$ stress and media lacking potassium. MS1 media contains $1 \%$ sucrose, 
a

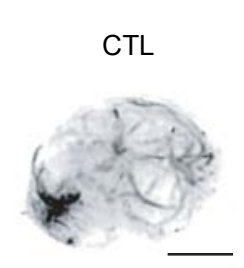

b

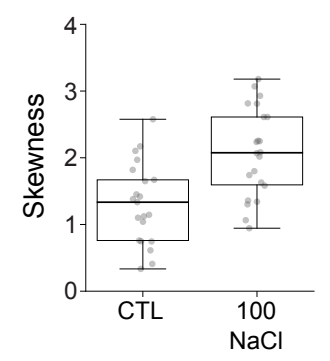

443

444

445

446

447

448

449

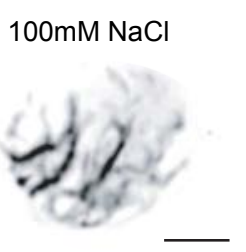

Extended Data 9. Salt stress promotes actin bundling in Chlamydomonas. a, Actin localization in Chlamydomonas cells under control conditions (CTL) and upon 16 hours treatment with $100 \mathrm{mM} \mathrm{NaCl}(\mathrm{NaCl})$. Actin filaments were visualized using Lifeact-NEOGREEN. Scale bar $=5 \mu \mathrm{m}$. b, Quantification of actin skewness in Chlamydomonas cells. 
a

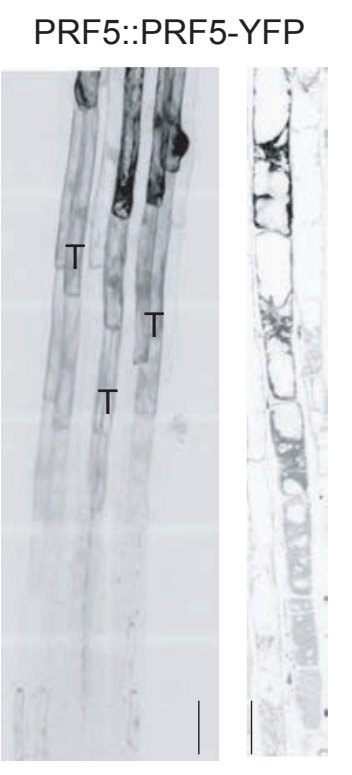

b Hours after transfer to $140 \mathrm{mM} \mathrm{NaCl}$

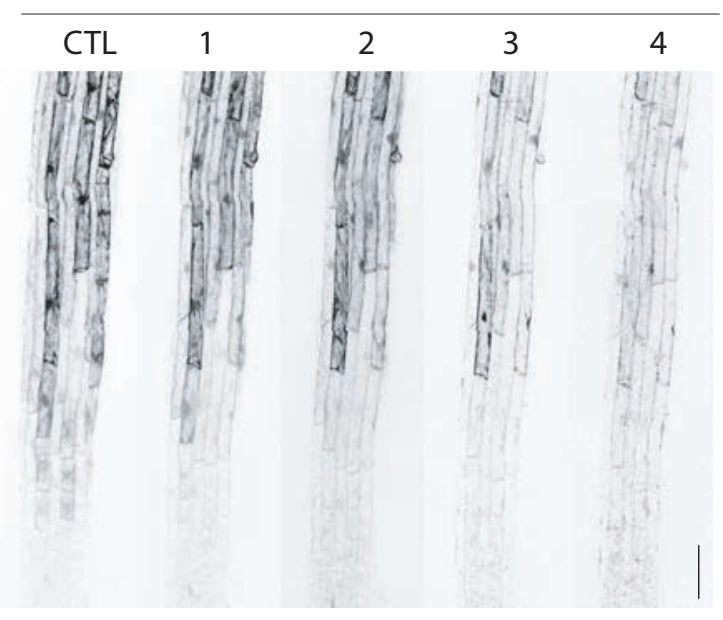

C Hours after transfer to $300 \mathrm{mM}$ Mannitol

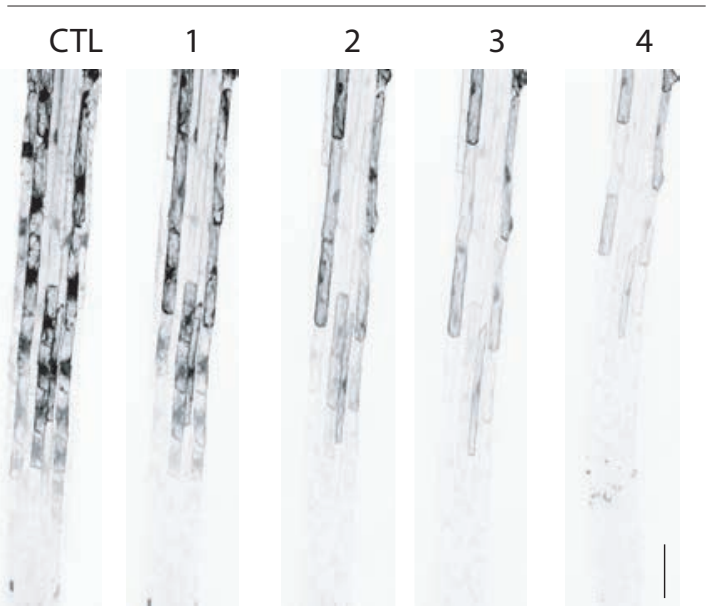

Extended Data 10. Profilin expression is restricted to root hairs and regulated by osmotic stress. a, Confocal image of Arabidopsis primary root elongation zone expressing ProPRF5::PRF5-YFP. T represents trichoblasts. Scale bar $=10 \mu \mathrm{m}$. b, Confocal images of Arabidopsis primary root elongation zone expressing ProPRF5::PRF5-YFP treated with $140 \mathrm{mM} \mathrm{NaCl}$ for the indicated hours. Scale bar $=20 \mu \mathrm{m}$. c, Confocal images of Arabidopsis primary root 


\section{List of supplementary materials}

461

462

Table S1| Chlamydomonas RNAseq raw data

463

Table S2| Chlamydomonas transcriptomic analysis

471

Table S3| Chlamydomonas phosphoproteomics raw data

473

474 Table S7| Chlamydomonas secondary screens

475

476

477

478

479

480

481

482

483

484

485

486

487

488

489

490

491

492

493

494

495

496

497

498 

All $C$. reinhardtii strains were maintained in Tris-Acetate-Phosphate (TAP) solid media, 1.5\% agar, with modified trace elements ${ }^{45}$ at $22{ }^{\circ} \mathrm{C}$ in low continuous light $\left(\sim 15-10 \mu \mathrm{mol}\right.$ photons $\mathrm{m}^{-2} \mathrm{~s}^{-}$ $\left.{ }^{1}\right)$. Lines harboring VENUS-3xFlagged tagged genes were maintained in the same conditions with media supplemented with $20 \mu \mathrm{g} / \mathrm{ml}$ hygromycin. Routine maintenance intervals were every four weeks.

\section{RNAseq samples and analysis}

RNA-seq samples: prior to sample collection wild-type strains (CC-4533) were refreshed in solid media and inoculated in liquid media, Tris-Acetate-Phosphate (TAP) with modified trace elements ${ }^{45}$ at $22{ }^{\circ} \mathrm{C}$ in low continuous light $(100 \mu \mathrm{mol}$ photons $\mathrm{m}-2 \mathrm{~s}-1)$. After 2 passages in liquid media ( $\sim 6$ doublings/passage), cells growing in early-mid exponential phase $(\sim 2 \mathrm{E}+06 \mathrm{cell} / \mathrm{s} / \mathrm{ml})$ were used for inoculation. Once cultures reached early-mid exponential phase $(\sim 2 \mathrm{E}+06$ cells $/ \mathrm{ml})$ different osmolyte concentrations were inoculated, $100 \mathrm{mM} \mathrm{NaCl}$ (Sigma) and $300 \mathrm{mM}$ mannitol using 1 liter Erlenmeyer flasks and grown with mild agitation $(\sim 150 \mathrm{rpm}), 22^{\circ} \mathrm{C}$ and low continuous light. Samples were collected at the designed timepoints from the same flask. Sample collection of $\sim 1 E 07$ cells/ml was performed using a funnel with a POLYVINYLIDENE FLUORIDE membrane (GCWP4700, millipore) coupled to a vacuum station and subsequently cells were snap-frozen in liquid nitrogen and saved at $-80^{\circ} \mathrm{C}$ until processing. For each treatment we performed 3 biological replicates with 2 control conditions, one at the beginning of the time course and another 24 hours after inoculation. RNA extraction was performed using Direct-zol RNA miniprep kit (R2050)) following manufacturer's instructions including in-column DNase I treatment prior RNA washing and elution steps. Each RNA sample was run on an Agilent 2100 Bioanalyzer RNA 6000 Nano chip for quantification and quality control. RNA samples were submitted to the Stanford Functional Genomics facility for subsequent library preparation and sequencing. RNA-seq libraries were made using the Kapa mRNA HyperPrep kit (Roche, KK8540) following manufacturer's protocols. Libraries were pooled based on fragment analyzer concentrations. Sequencing was performed on Nextseq high-output flow cell, $1 \times 75$ bp run (Ilumina). RNA-seq reads were aligned using BWA to the Chlamydomonas reference genome $5.6^{46}$. DESeq2 package was used to call transcripts as differentially expressed at a false-discovery-rate (Benjamini-Hochberg method) $\mathrm{FC}>2$, (FDR $<0.01$ ). GO-term enrichment analysis was performed with the BINGO plugin for Cytoscape ${ }^{47}$. To perform GO-term-enrichment analysis of differentially regulated transcripts, we used several approaches. First, we used GO terms from the Joint Genome Institute (JGI) for Chlamydomonas genome 5.6 version, and used these as a reference set. Second, we used the annotations of the homologous genes in Arabidopsis thaliana. To identify these homologues, we identified gene pairs by reciprocal best BLAST hit (RBH) and 
selected "Best hit" for each pair having the lowest e-value (Onishi 2018), setting an e-value threshold of 1E-10.

\section{Proline quantification}

Proline quantification in Chlamydomonas cells was adapted from ${ }^{48}$. Briefly, 1E05 cells from control or treated samples in early-mid exponential growing phase were collected and transferred to 96 wells plates together with L-proline standards. Subsequently the same volume of glacial acetic acid and acid ninhydrin was added to each well, sealed and incubated for 1 hour at $100{ }^{\circ} \mathrm{C}$. Following incubation and cool down of samples chromophore was extracted with Toluene and the absorbance read at $520 \mathrm{~nm}$ using toluene as blank. Determination of proline concentration was performed from a standard curve.

\section{Phosphoproteomics samples and analysis}

Phosphoproteomics samples: prior to sample collection wild-type strains (CMJ030/CC-4533) were refreshed in solid media and inoculated in liquid media, Tris-Acetate-Phosphate (TAP) with modified trace elements ${ }^{45}$ at $22{ }^{\circ} \mathrm{C}$ in low continuous light $(100 \mu \mathrm{mol}$ photons $\mathrm{m}-2 \mathrm{~s}-1)$. After 2 passages of cells growing in early-mid exponential phase ( $\sim 6$ doublings/passage), with either $14 \mathrm{NH}_{4} \mathrm{Cl}$ or $15 \mathrm{NH}_{4} \mathrm{Cl}$ as a sole source of nitrogen, cells growing in early-mid exponential phase $(\sim 2 \mathrm{E}+06$ cells $/ \mathrm{ml})$ were used to inoculate 2-liter bottles with bubbled air and constant stirring at an initial concentration of $2 \mathrm{E} 04$ cells $/ \mathrm{ml}$. This ensured $15 \mathrm{~N}$ growth for at least eight generations. Cells at early-mid exponential phase were spun out $\left(2,000 \mathrm{~g}, 5 \mathrm{~min}, 4^{\circ} \mathrm{C}\right)$ and resuspended in a $1: 1$ $(\mathrm{v} / \mathrm{w})$ ratio of ice cold homogenization buffer (290 mM sucrose, $250 \mathrm{mM}$ Tris-HCL $(\mathrm{pH}=8), 25$ mM EDTA, $25 \mathrm{mM}$ sodium fluoride, $50 \mathrm{mM}$ sodium pyrophosphate, $1 \mathrm{mM}$ ammonium molybdate, $0.5 \%$ polyvinyl pyrovinyl pyrrolidone in water and 1 cOmplete EDTA-free protease inhibitor (Sigma-Aldrich) $/ 50 \mathrm{ml}$ ). The cell slurry was then added drop wise to liquid nitrogen to form small Chlamydomonas pellets approximately $5 \mathrm{~mm}$ in diameter. Samples were stored at $-80{ }^{\circ} \mathrm{C}$. with minor modifications. Samples were combined in an experimental pair consisting of one treated sample grown in $14 \mathrm{NH}_{4} \mathrm{Cl}$ and one control sample growing in $15 \mathrm{NH}_{4} \mathrm{Cl}$. For the second reciprocal experimental pair, the samples were combined in the inverse fashion $\left(15 \mathrm{NH}_{4} \mathrm{Cl}\right.$ treated and $14 \mathrm{NH}_{4} \mathrm{Cl}$ control). Frozen cells were combined at 1:1 weight ratio prior homogenization, with a total weight of 4 grams ( 2 grams of cells grown in $14 \mathrm{NH}_{4} \mathrm{Cl}$ and 2 grams of $15 \mathrm{NH}_{4} \mathrm{CL}$ ). Three biological replicates were processed for each treatment condition. For all experiments, samples were processed in homogenization buffer supplemented with phosphatase inhibitors using a sonicator ( $1 \mathrm{~cm}$ probe, $(12 \times 5$ seconds) x2, and 50\% duty cycle (Bransom 45 Digital Sonifier (Marshall Scientific)) while kept on ice. The resulting homogenate was filtered through two layers of Miracloth (Calbiochem) and spun out 15 minutes at 1,500 g and $4{ }^{\circ} \mathrm{C}$. Subsequent steps were performed as described previously ${ }^{49}$. 
Chlamydomonas reinhardt annotation v5.6 protein database using MASCOT software (Matrix MASCOT search results were filtered to maintain a $1 \%$ false discovery rate at the peptide level using a reverse-protein sequence database. Quantitative ratio measurements from peak areas were performed using Census software. Only phosphopeptides showing reciprocal changes of 2-fold or greater in two of the three replicates were selected for data shown in Figure 1. Phosphopeptides showing reciprocal changes of 1.5 -fold or greater were used to identify homologous phosphorylated proteins in Arabidopsis (Extended Data 3).

\section{Chlamydomonas mutant screen} library preparation were performed as described ${ }^{50}$. We used several approaches to identify mutants with growth defects, as described ${ }^{50}$. High-confidence gene-phenotype relationships (Figure 2, Extended Data 6, Table S4) were based on false discovery rate (FDR $<0.3$ ) performed on p-values of genes with more than 2 alleles using the Benjamini-Hochberg method ${ }^{51}$.

\section{Secondary screens}

Chlamydomonas mutants selected for secondary screens were picked from the Chlamydomonas mutant library and inoculated in 96-well plates containing liquid TAP and $10 \mu \mathrm{g} / \mathrm{ml}$ paromomycin. Plates were re-inoculated 3 times (ensuring $\sim 10$ doublings), and early-mid exponential cells were used to inoculate control plates (TAP liquid), plates supplemented with $100 \mathrm{mM} \mathrm{NaCl}$ or plates containing $300 \mathrm{mM}$ mannitol. Plates were grown under low light conditions. 5 days postinoculation plates were scanned using a CanonScan 9000F flatbed scanner (Canon). Images were quantified using Fiji. 8-bit RGB images were converted to gray scale and average pixel intensity was measured with a sampling area including the entire well. Wells with bubbles and/or irregular clamping were avoided and not quantified. Comparisons between average pixel intensity were used to estimate the mutant growth compared to wild type. Each plate contained 2 biological replicates of each mutant and multiple wild-type colonies to avoid any positional effect. The resulting Z-scores can be found in Table S7.

\section{Chlamydomonas protein localization}

To generate fluorescently tagged proteins in Chlamydomonas, we used pRAM118 (a generous gift from Silvia Ramundo). Open reading frames were PCR amplified (Phusion Hotstart II polymerase, ThermoFisher Scientific or KOD DNA polymerase, Millipore) from genomic DNA or BAC, gel purified (MinElute Gel Extraction Kit, QIAGEN) and cloned in frame with the C-terminal VENUS-3xFLAG tag by Gibson assembly. Primers were designed to amplify target genes from their predicted start codon up to, but not including the stop codon. All resulting constructs were verified by Sanger sequencing. Constructs were linearized by EcoRV prior to transformation. Electroporation was performed with cells grown to $\sim 8 \mathrm{E} 06 \mathrm{cells} / \mathrm{ml}$, resuspended at $\sim 8 \mathrm{E} 08 \mathrm{cells} / \mathrm{ml}$ in CHES buffer at room temperature, and electroporated in a volume of $125 \mu 1$ in a 2-mm-gap 
electro cuvette using a NEPA21 square-pulse electroporator (Bulldog Bio), using two poring pulses of 250 and $150 \mathrm{~V}$ for $8 \mathrm{msec}$ each, and five transfer pulses of $50 \mathrm{msec}$ each starting at $20 \mathrm{~V}$ with a "decay rate" of $40 \%{ }^{52}$. Cells were transferred to a $15-\mathrm{ml}$ centrifugation tube containing 8 $\mathrm{ml}$ TAP plus $40 \mathrm{mM}$ sucrose. After overnight incubation at $24{ }^{\circ} \mathrm{C}$ under low light, cells were collected by centrifugation and spread on TAP agar $(1.5 \% \mathrm{w} / \mathrm{v})$ plates containing $25 \mu \mathrm{g} / \mathrm{ml}$

624 hygromycin.

625 Colonies for plate reader analysis resulting from transformation plates were picked and transferred 626 to a 96-well microplate plate containing $150 \mu \mathrm{l}$ liquid of TAP supplemented with $25 \mu \mathrm{g} / \mathrm{ml}$ hygromycin, without shaking. Fluorescence readings were acquired with excitation and emission wavelengths of 515 and $550 \mathrm{~nm}$, TECAN infinite 200 Pro microplate reader. Five independent colonies with values greater than background were further selected for imaging. Expression of Cre12.g524950-VENUS (LEO) was further confirmed with Western blot.

\section{Arabidopsis growth and treatments}

Arabidopsis thaliana Col-0 ecotype was used in this study. A complete list of the mutant lines used in this study can be found Table S9. Seeds were surface sterilized by washing with $20 \%$ bleach for 5 minutes and rinsed with sterile deionized water four times. After stratification seeds were grown in $10 \times 10 \mathrm{~cm}$ petri dish plates containing sterile full MS media (MSP01-50LT; Caisson), 1\% Sucrose (Sigma-Aldrich), 0.7\% Gelzan, 0.05\% MES (Sigma-Aldrich) adjusted to $\mathrm{pH}=5.7 \mathrm{using}$ $1 \mathrm{M} \mathrm{KOH}$. Screening of Arabidopsis mutants was performed with seedlings growing under standard media and transferred to plates containing $140 \mathrm{mM} \mathrm{NaCl}$ (Sigma-Aldrich) or $300 \mathrm{mM}$ mannitol (Sigma-Aldrich) after 4 days post germination. with $14 \mathrm{~h}$ light and $10 \mathrm{~h}$ dark cycles at $130 \mu \mathrm{mol} \mathrm{m} \mathrm{m}^{-1}$ light intensity. Plates were sealed using micropore tape $(3 \mathrm{M})$. Plates were placed vertically to allow vertical growth of the roots. Images of seedlings were captured using Epson perfection V800 Photo color scanner, root length was quantified using Fiji ${ }^{53}$.

648 Confocal laser microscopy was performed on a Leica TCS SP8 inverted confocal scanning 649 microscope in resonant scanning mode with LASX software. Chlamydomonas cells were mounted in chambered coverglass (Ibidi, 80826), covered with 2\% low melting agar and imaged with 93x/1.3 NA glycerin immersion objective. Arabidopsis roots were mounted in chambered coverglass (ThermoFisher, 155360) with a pad of Gelzan MS media or gelzan MS media supplemented with corresponding treatment. Root growth time lapse movies were taken using a 20x/0.75 NA glycerin immersion objective. For each root 5 tiles were taken using the Navigator mode, with the root tip position in the middle of the second tile at the beginning of the time course, to ensure capture of the root growth upon the 16 hour period. Fluorescence of LTi6b-YFP, ABD2detection from 500-550 nm with a HyD SMD hybrid detector (Leica). 
659 All image quantifications were performed with Fiji ${ }^{53}$. Quantification of root cell death was

660 performed using Z-stack projections and manual counting of cell death. Actin quantification was

661 performed with stack images taken from the first visual cortical actin in the epidermal elongating

662 cells, collecting 20 steps of $1 \mu \mathrm{m}$ each. Imaging parameters gain and pinhole were selected such

663 that individual actin filaments could be observed, but actin filament bundles were not saturated.

664 All cropped images used for quantification were taken from original 8-bit files. Actin images were

665 cropped along the entire length of every cell in the elongation zone. Skewness was analyzed

666 according to ${ }^{54}$. No image processing was applied to maximum-intensity projections that were

667 analyzed for skewness. The size of each cell crop was maintained constant across all images and

668 was smaller than the entire length of a cell $(10 \times 10 \mu \mathrm{m})$. Relative angle of actin filaments was

669 computed using Fiji and aligning cells horizontally. Calcium spikes quantification was performed

670 at the transition zone in root epidermal cells $(\sim 200 \mu \mathrm{m}$ from the stem cell niche) during a period

671 of 4 hours. Registration was applied to time lapses using the HyperStackReg plugin. A

672 MaxEntropy Threshold was applied, and 8 ROIs $(24 \times 24 \mu \mathrm{m})$ were selected across a $150 \mu \mathrm{m}$ area

673 to measure the signal corresponding to calcium spikes. A minimum of 6 pixels was used to exclude

674 sporadic signals. Calcium spikes and their duration were confirmed manually.

675

676 Data availability

677 Strains, plasmids and plasmid sequences are available upon request and will be deposited at the 678 Chlamydomonas Resource Centre. 


\section{References}

681 1. Hohmann, S. Osmotic stress signaling and osmoadaptation in yeasts. Microbiol. Mol. Biol. Rev. 66, 300-372 (2002).

2. van Zelm, E., Zhang, Y. \& Testerink, C. Salt Tolerance Mechanisms of Plants. Annu. Rev. Plant Biol. 71, 403-433 (2020).

3. Mackinder, L. C. M. et al. A Spatial Interactome Reveals the Protein Organization of the Algal CO2Concentrating Mechanism. Cell 171, 133-147.e14 (2017).

4. Saito, H. \& Posas, F. Response to hyperosmotic stress. Genetics 192, 289-318 (2012).

5. Dinneny, J. R. et al. Cell identity mediates the response of Arabidopsis roots to abiotic stress. Science 320, 942-945 (2008).

6. Merchant, S. S. et al. The Chlamydomonas genome reveals the evolution of key animal and plant functions. Science 318, 245-250 (2007).

7. Gonzalez-Guzman, M. et al. Arabidopsis PYR/PYL/RCAR receptors play a major role in quantitative regulation of stomatal aperture and transcriptional response to abscisic acid. Plant Cell 24, 2483-2496 (2012).

8. Geng, Y. et al. A spatio-temporal understanding of growth regulation during the salt stress response in Arabidopsis. Plant Cell 25, 2132-2154 (2013).

9. Pazour, G. J., Agrin, N., Leszyk, J. \& Witman, G. B. Proteomic analysis of a eukaryotic cilium. J. Cell Biol. 170, 103-113 (2005).

10. Zhou, H. et al. Ubiquitin-specific protease 16 modulates salt tolerance in Arabidopsis by regulating $\mathrm{Na}(+) / \mathrm{H}(+)$ antiport activity and serine hydroxymethyltransferase stability. Plant Cell 24, 5106-5122 (2012).

11. Baek, D. et al. The GIGANTEA-ENHANCED EM LEVEL Complex Enhances Drought Tolerance via Regulation of Abscisic Acid Synthesis. Plant Physiol. 184, 443-458 (2020).

12. Finkelstein, R. R. \& Lynch, T. J. The Arabidopsis abscisic acid response gene ABI5 encodes a basic leucine zipper transcription factor. Plant Cell 12, 599-609 (2000).

13. Ando, A., Tanaka, F., Murata, Y., Takagi, H. \& Shima, J. Identification and classification of genes required for tolerance to high-sucrose stress revealed by genome-wide screening of Saccharomyces cerevisiae. FEMS Yeast Res. 6, 249-267 (2006).

14. Lee, E. et al. Ionic stress enhances ER-PM connectivity via phosphoinositide-associated SYT1 contact site expansion in Arabidopsis. Proc. Natl. Acad. Sci. U. S. A. 116, 1420-1429 (2019).

15. Bonangelino, C. J. et al. Osmotic stress-induced increase of phosphatidylinositol 3,5-bisphosphate requires Vac14p, an activator of the lipid kinase Fab1p. J. Cell Biol. 156, 1015-1028 (2002).

16. Li, X. et al. A genome-wide algal mutant library and functional screen identifies genes required for eukaryotic photosynthesis. Nat. Genet. 51, 627-635 (2019).

17. Yuan, F. et al. OSCA1 mediates osmotic-stress-evoked Ca2+ increases vital for osmosensing in Arabidopsis. Nature 514, 367-371 (2014).

18. Hamilton, E. S. et al. Mechanosensitive channel MSL8 regulates osmotic forces during pollen hydration and germination. Science 350, 438-441 (2015).

19. Yuan, F. et al. OSCA1 mediates osmotic-stress-evoked Ca2+ increases vital for osmosensing in Arabidopsis. Nature 514, 367-371 (2014).

20. Haswell, E. S. \& Meyerowitz, E. M. MscS-like proteins control plastid size and shape in Arabidopsis thaliana. Curr. Biol. 16, 1-11 (2006).

21. Yokoi, S. et al. Differential expression and function of Arabidopsis thaliana $\mathrm{NHX} \mathrm{Na/H}$ antiporters in the salt stress response. Plant J. 30, 529-539 (2002).

22. Nass, R. \& Rao, R. Novel localization of a $\mathrm{Na}+/ \mathrm{H}+$ exchanger in a late endosomal compartment of yeast. Implications for vacuole biogenesis. J. Biol. Chem. 273, 21054-21060 (1998).

23. Liu, W.-C. et al. WD40-REPEAT 5a functions in drought stress tolerance by regulating nitric oxide accumulation in Arabidopsis. Plant Cell Environ. 40, 543-552 (2017).

24. Bittner, F., Oreb, M. \& Mendel, R. R. ABA3 Is a Molybdenum Cofactor Sulfurase Required for Activation of 

(2001).

25. Xiong, L., Lee, H., Ishitani, M. \& Zhu, J.-K. Regulation of Osmotic Stress-responsive Gene Expression by theLOS6/ABA1 Locus inArabidopsis *. J. Biol. Chem. 277, 8588-8596 (2002).

26. Evans, T. G. Considerations for the use of transcriptomics in identifying the 'genes that matter' for environmental adaptation. J. Exp. Biol. 218, 1925-1935 (2015).

27. Giaever, G. et al. Functional profiling of the Saccharomyces cerevisiae genome. Nature 418, 387-391 (2002).

28. Gutman, B. L. \& Niyogi, K. K. Chlamydomonas and Arabidopsis. A dynamic duo. Plant Physiol. 135, 607610 (2004).

29. Cutler, S. R., Ehrhardt, D. W., Griffitts, J. S. \& Somerville, C. R. Random GFP::cDNA fusions enable visualization of subcellular structures in cells of Arabidopsis at a high frequency. Proc. Natl. Acad. Sci. U.S. A. 97, 3718-3723 (2000).

30. Porta, J. C. \& Borgstahl, G. E. O. Structural basis for profilin-mediated actin nucleotide exchange. J. Mol. Biol. 418, 103-116 (2012).

31. Funk, J. et al. Profilin and formin constitute a pacemaker system for robust actin filament growth. Elife 8, (2019).

32. Sheahan, M. B., Staiger, C. J., Rose, R. J. \& McCurdy, D. W. A green fluorescent protein fusion to actinbinding domain 2 of Arabidopsis fimbrin highlights new features of a dynamic actin cytoskeleton in live plant cells. Plant Physiol. 136, 3968-3978 (2004).

33. Arieti, R. S. \& Staiger, C. J. Auxin-induced actin cytoskeleton rearrangements require AUX1. New Phytol. 226, 441-459 (2020).

34. Choi, W.-G., Toyota, M., Kim, S.-H., Hilleary, R. \& Gilroy, S. Salt stress-induced Ca2+ waves are associated with rapid, long-distance root-to-shoot signaling in plants. Proc. Natl. Acad. Sci. U. S. A. 111, 6497-6502 (2014).

35. Feng, W. et al. The FERONIA Receptor Kinase Maintains Cell-Wall Integrity during Salt Stress through Ca2+ Signaling. Curr. Biol. 28, 666-675.e5 (2018).

36. Papakonstanti, E. A. \& Stournaras, C. Cell responses regulated by early reorganization of actin cytoskeleton. FEBS Lett. 582, 2120-2127 (2008).

37. Endler, A. et al. A Mechanism for Sustained Cellulose Synthesis during Salt Stress. Cell 162, 1353-1364 (2015).

38. Zwiewka, M., Nodzyński, T., Robert, S., Vanneste, S. \& Friml, J. Osmotic Stress Modulates the Balance between Exocytosis and Clathrin-Mediated Endocytosis in Arabidopsis thaliana. Mol. Plant 8, 1175-1187 (2015).

39. Gutierrez, R., Lindeboom, J. J., Paredez, A. R., Emons, A. M. C. \& Ehrhardt, D. W. Arabidopsis cortical microtubules position cellulose synthase delivery to the plasma membrane and interact with cellulose synthase trafficking compartments. Nat. Cell Biol. 11, 797-806 (2009).

40. Tardif, M. et al. PredAlgo: a new subcellular localization prediction tool dedicated to green algae. Mol. Biol. Evol. 29, 3625-3639 (2012).

41. Urzica, E. I. et al. Systems and trans-system level analysis identifies conserved iron deficiency responses in the plant lineage. Plant Cell 24, 3921-3948 (2012).

42. Jo, W. J. et al. Novel insights into iron metabolism by integrating deletome and transcriptome analysis in an iron deficiency model of the yeast Saccharomyces cerevisiae. BMC Genomics 10, 130 (2009).

43. Bhaskara, G. B., Nguyen, T. T., Yang, T.-H. \& Verslues, P. E. Comparative Analysis of Phosphoproteome Remodeling After Short Term Water Stress and ABA Treatments versus Longer Term Water Stress Acclimation. Front. Plant Sci. 8, 523 (2017).

44. Wang, P. et al. Quantitative phosphoproteomics identifies SnRK2 protein kinase substrates and reveals the effectors of abscisic acid action. Proc. Natl. Acad. Sci. U. S. A. 110, 11205-11210 (2013).

45. Kropat, J. et al. A revised mineral nutrient supplement increases biomass and growth rate in Chlamydomonas reinhardtii. Plant J. 66, 770-780 (2011). 
46. Li, H. \& Durbin, R. Fast and accurate short read alignment with Burrows-Wheeler transform. Bioinformatics 25, 1754-1760 (2009).

47. Maere, S., Heymans, K. \& Kuiper, M. BiNGO: a Cytoscape plugin to assess overrepresentation of Gene Ontology categories in Biological Networks. Bioinformatics 21, 3448-3449 (2005).

48. Bates, L. S., Waldren, R. P. \& Teare, I. D. Rapid determination of free proline for water-stress studies. Plant Soil 39, 205-207 (1973).

49. Minkoff, B. B., Burch, H. L. \& Sussman, M. R. A Pipeline for 15N Metabolic Labeling and Phosphoproteome Analysis in Arabidopsis thaliana. in Arabidopsis Protocols (eds. Sanchez-Serrano, J. J. \& Salinas, J.) 353-379 (Humana Press, 2014).

50. Vilarrasa-Blasi, J. et al. Systematic characterization of gene function in a photosynthetic organism. Cold Spring Harbor Laboratory 2020.12.11.420950 (2020) doi:10.1101/2020.12.11.420950.

51. Benjamini, Y. \& Hochberg, Y. Controlling the false discovery rate: A practical and powerful approach to multiple testing. J. R. Stat. Soc. 57, 289-300 (1995).

52. Onishi, M. \& Pringle, J. R. Robust Transgene Expression from Bicistronic mRNA in the Green Alga Chlamydomonas reinhardtii. G3 6, 4115-4125 (2016).

53. Schindelin, J. et al. Fiji: an open-source platform for biological-image analysis. Nat. Methods 9, 676-682 (2012).

54. Higaki, T., Kutsuna, N., Sano, T., Kondo, N. \& Hasezawa, S. Quantification and cluster analysis of actin cytoskeletal structures in plant cells: role of actin bundling in stomatal movement during diurnal cycles in Arabidopsis guard cells. Plant J. 61, 156-165 (2010). 